DFT Investigation of the Single-Center, Two-State Model for the Broken Rate Order of Transition Metal Catalyzed Olefin Polymerization

Vidar R. Jensen, ${ }^{* \dagger}$ Debasis Koley ${ }^{\dagger}$, Mavinahalli N. Jagadeesh ${ }^{\dagger}$ and Walter Thiel ${ }^{*}, \dagger$ Department of Chemistry, University of Bergen, Allégaten 41, N-5007 Bergen, Norway, and Max-Planck-Institut für Kohlenforschung, Kaiser-Wilhelm-Platz 1, D-45470 Mülheim an der Ruhr, Germany

‡University of Bergen. E-mail: Vidar.Jensen@kj.uib.no.

${ }^{\dagger}$ Max-Planck-Institut für Kohlenforschung. E-mail: thiel@mpi-muelheim.mpg.de.

Supporting Information (for 37 pages).

\title{
Contents:
}

Table S1 Total energies, enthalpies and Gibbs free energies (a.u.) as obtained in the DFT calculations.

Table S2 Cartesian coordinates of minima and transition states given in angstroms. 
Table S1. Total energies, enthalpies and Gibbs free energies (a.u.) as obtained in the DFT calculations.

\begin{tabular}{|c|c|c|c|}
\hline Entry & $\mathrm{E}_{\mathrm{e}}$ & $\mathrm{H}_{298}$ & $\mathrm{G}_{298}$ \\
\hline I $\beta 1$ & -552.0696211 & -551.798686 & -551.857254 \\
\hline $\mathbf{I} \alpha 1$ & -552.055856 & -551.784472 & -551.843770 \\
\hline $\mathbf{I} \gamma \mathbf{1}$ & -552.062115 & -551.790294 & -551.848070 \\
\hline $\mathrm{I} \alpha 2$ & -552.055200 & -551.783793 & -551.842855 \\
\hline IH1 & -552.053657 & -551.785427 & -551.842493 \\
\hline IH2 & -552.054433 & -551.787640 & -551.843901 \\
\hline IA & -550.866581 & -550.617255 & -550.673988 \\
\hline ISß2 & -552.066324 & -551.796328 & -551.852454 \\
\hline IS及1 & -552.064845 & -551.793766 & -551.852619 \\
\hline$[\mathbf{I} \boldsymbol{\beta} 1-\mathbf{I} \alpha \mathbf{1}]^{\neq}$ & -552.051061 & -551.780905 & -551.840301 \\
\hline$[\mathbf{I} \gamma \mathbf{1}-\mathbf{I} \boldsymbol{\alpha} \mathbf{1}]^{\neq}$ & -552.050466 & -551.780465 & -551.839428 \\
\hline$[\mathbf{I} \boldsymbol{\gamma} \mathbf{1}-\mathbf{I} \boldsymbol{\beta} 1]^{\neq}$ & -552.055795 & -551.784818 & -551.841570 \\
\hline$[\mathbf{I} \alpha 1-\mathbf{I} \alpha 2]^{\neq}$ & -552.051713 & -552.781363 & -551.838878 \\
\hline$[\mathbf{I} \alpha \mathbf{1}-\mathbf{I F} \alpha \mathbf{1}]^{\neq}$ & -630.663707 & -630.337439 & -630.411058 \\
\hline$[\mathbf{I} \beta 1-\mathrm{IF} \beta 1]^{\neq}$ & -630.675125 & -630.349250 & -630.425417 \\
\hline$[\mathbf{I} \gamma \mathbf{1}-\mathbf{I F} \boldsymbol{\alpha} 2]^{\neq}$ & -630.665049 & -630.337830 & -630.405383 \\
\hline IF $\alpha 1$ & -630.681542 & -630.353550 & -630.421433 \\
\hline IFa2 & -630.673296 & -630.345312 & -630.412718 \\
\hline IF $\beta 1$ & -630.684595 & -630.356035 & -630.421444 \\
\hline$[\mathrm{IF} \boldsymbol{\beta} 1-\mathrm{IF} \alpha 1]^{\neq}$ & -630.678413 & -630.351322 & -630.417340 \\
\hline$[\mathbf{I F} \boldsymbol{\alpha} \mathbf{1}-\mathbf{I P} \boldsymbol{\gamma}]^{\neq}$ & -630.677461 & -630.350736 & -630.417125 \\
\hline $\mathbf{I P \gamma}$ & -630.703925 & -630.373742 & -630.438195 \\
\hline$[\mathbf{I} \alpha 2-I B \alpha 2]^{\neq}$ & -630.658311 & -630.332472 & -630.405533 \\
\hline $\mathrm{IB} \boldsymbol{\alpha} 2$ & -630.676181 & -630.347755 & -630.414097 \\
\hline$[\mathrm{IB} \boldsymbol{\alpha} 2-\mathrm{IP} \gamma]^{\neq}$ & -630.674624 & -630.347921 & -630.413245 \\
\hline IIß1 & -945.191458 & -944.629483 & -944.723168 \\
\hline II $\gamma 1$ & -945.182300 & -944.619981 & -944.713013 \\
\hline $\mathrm{II} \alpha 2$ & -945.177320 & -944.615774 & -944.713084 \\
\hline IIS $\beta 1$ & -945.183826 & -944.621931 & -944.714942 \\
\hline IIS $\beta 2$ & -945.182343 & -944.621842 & -944.713467 \\
\hline IIA & -943.987798 & -943.448257 & -943.541126 \\
\hline IIH2 & -945.170298 & -944.613225 & -944.704788 \\
\hline IIH1 & -945.170123 & -944.611639 & -944.707814 \\
\hline$[\mathrm{II} \beta 1-\mathrm{II} \alpha 2]^{\neq}$ & -945.175106 & -944.614513 & -944.709990 \\
\hline$[\mathrm{II} \gamma 1-\mathrm{II} \alpha 2]^{\neq}$ & -945.176350 & -944.615329 & -944.710541 \\
\hline$[\mathbf{I I} \gamma \mathbf{1}-\mathrm{II} \boldsymbol{\beta} 1]^{\neq}$ & -945.175277 & -944.613792 & -944.706362 \\
\hline$[\mathrm{II} \beta 1-\mathrm{IIF} \beta 1]^{\neq}$ & -1023.785656 & -1023.167954 & -1023.268529 \\
\hline$[\mathrm{II} \alpha 2-\mathrm{IIIP} \gamma]^{\neq}$ & -1023.775132 & -1023.158580 & -1023.263604 \\
\hline IIF $\alpha 1$ & -1023.787908 & -1023.1695 & -1023.271499 \\
\hline IIF $\beta 1$ & -1023.788002 & -1023.169110 & -1023.267940 \\
\hline IIF $\alpha 2$ & -1023.780374 & -1023.16187 & -1023.263148 \\
\hline$[\mathrm{II} \alpha 2-\mathrm{IIF} \alpha 1]^{\neq}$ & -1023.78226 & -1023.165258 & -1023.272122 \\
\hline
\end{tabular}




\begin{tabular}{llll}
$\mathbf{[ I I F} \boldsymbol{\alpha} \mathbf{1}-\mathbf{I I P} \boldsymbol{\gamma}]^{\neq}$ & -1023.78109 & -1023.1637 & -1023.262356 \\
$\mathbf{I I P} \boldsymbol{\gamma}$ & -1023.821675 & -1023.200860 & -1023.300934 \\
$\mathbf{I} \boldsymbol{\alpha} \mathbf{1}-\mathbf{M}$ & -1417.859763 & -1417.15942 & -1417.308129 \\
$\mathbf{I A - M}$ & -1416.646813 & -1415.96821 & -1416.112913 \\
\hline
\end{tabular}

Table S2 Cartesian coordinates of minima and transition states given in angstroms. 


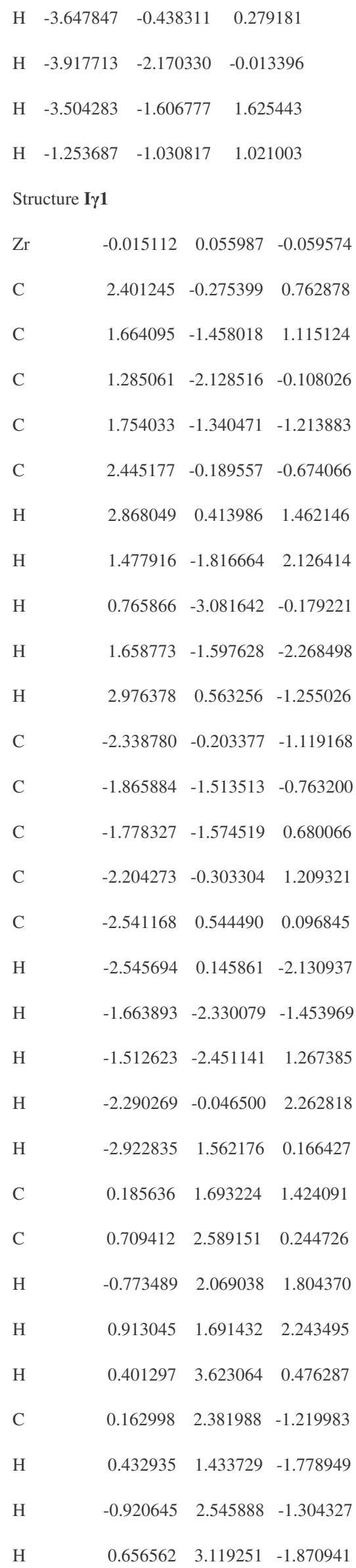

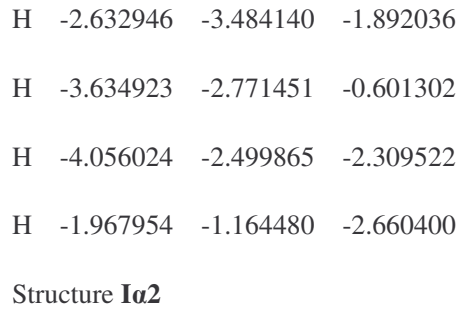


$\mathrm{H}$

$\begin{array}{lll}1.807747 & 2.583408 & 0.207147\end{array}$

Structure IH1

$\begin{array}{llll}\mathrm{Zr} & 0.157916 & 0.003736 & -0.031648\end{array}$

$\begin{array}{lllll}\text { C } & -0.695431 & -0.086211 & 2.379768\end{array}$

$\begin{array}{llll}\text { C } & 0.688506 & -0.512346 & 2.389279\end{array}$

$\begin{array}{llll}\text { C } & 1.508230 & 0.616480 & 2.027145\end{array}$

$\begin{array}{llll}\text { C } & 0.627495 & 1.724700 & 1.752610\end{array}$

$\begin{array}{lllll}\text { C } & & -0.732603 & 1.289323 & 1.976779\end{array}$

$\begin{array}{llll}\text { C } & -1.394177 & 0.278771 & -2.058405\end{array}$

$\begin{array}{lllll}\text { C } & & -1.498877 & 1.463769 & -1.256462\end{array}$

$\begin{array}{lllll}\text { C } & & -0.215060 & 2.136191 & -1.291025\end{array}$

$\begin{array}{llll}\text { C } & 0.678345 & 1.360098 & -2.110652\end{array}$

$\begin{array}{lllll}\text { C } & -0.044854 & 0.196126 & -2.564024\end{array}$

$\begin{array}{llll}\text { C } & -1.202681 & -2.448492 & 0.309198\end{array}$

$\begin{array}{llll}\text { C } & -0.285752 & -2.481544 & -0.707931\end{array}$

$\begin{array}{llll}\mathrm{H} & -0.908737 & -2.627173 & 1.345261\end{array}$

$\begin{array}{llll}\mathrm{H} & -2.274428 & -2.401490 & 0.095845\end{array}$

$\begin{array}{llll}\mathrm{H} & -0.650011 & -2.460058 & -1.738980\end{array}$

$\begin{array}{lllll}\text { C } & & 1.199736 & -2.540505 & -0.507045\end{array}$

$\begin{array}{llll}\mathrm{H} & 1.700739 & -3.052286 & -1.342130\end{array}$

$\begin{array}{lllll}\mathrm{H} & 1.725775 & -1.513043 & -0.530471\end{array}$

$\begin{array}{llll}\mathrm{H} & 1.948571 & 0.213544 & -0.346589\end{array}$

$\begin{array}{llll}\mathrm{H} & 1.487272 & -3.003272 & 0.446589\end{array}$

$\begin{array}{lllll}\mathrm{H} & -2.402919 & 1.832441 & -0.775867\end{array}$

$\begin{array}{llll}\mathrm{H} & -2.204790 & -0.416998 & -2.270088\end{array}$

$\begin{array}{llll}\mathrm{H} & 0.007921 & 3.103050 & -0.846687\end{array}$

$\begin{array}{llll}\mathrm{H} & 1.695772 & 1.628251 & -2.382996\end{array}$

$\begin{array}{llll}\mathrm{H} & 0.343136 & -0.561972 & -3.242813\end{array}$

$\begin{array}{llll}\text { H } & 0.943838 & 2.732453 & 1.492985\end{array}$

$\begin{array}{llll}\mathrm{H} & -1.622211 & 1.912319 & 1.911886\end{array}$

$\begin{array}{llll}\mathrm{H} & -1.556561 & -0.677180 & 2.685595\end{array}$

$\begin{array}{llll}\mathrm{H} & 1.058194 & -1.483596 & 2.714606\end{array}$

$\begin{array}{llll}\mathrm{H} & 2.594554 & 0.647428 & 2.014359\end{array}$

Structure IA

$\mathrm{Zr} \quad 0.072331 \quad 0.002526 \quad-0.007923$
H $\quad 2.033853 \quad 1.361536 \quad 1.424229$

Structure IH2

\begin{tabular}{|c|c|c|c|}
\hline $\mathrm{Zr}$ & 0.169210 & -0.032323 & -0.034051 \\
\hline $\mathrm{C}$ & -0.827383 & -0.096076 & 2.316409 \\
\hline $\mathrm{C}$ & 0.506763 & -0.635269 & 2.463058 \\
\hline $\mathrm{C}$ & 1.445066 & 0.409725 & 2.177296 \\
\hline $\mathrm{C}$ & 0.701585 & 1.597531 & 1.825356 \\
\hline $\mathrm{C}$ & -0.703548 & 1.286204 & 1.940226 \\
\hline $\mathrm{C}$ & -1.377392 & 0.068625 & -2.080106 \\
\hline $\mathrm{C}$ & -1.756725 & 1.135908 & -1.194707 \\
\hline $\mathrm{C}$ & -0.667657 & 2.087656 & -1.152942 \\
\hline C & 0.387733 & 1.592761 & -1.990468 \\
\hline C & -0.039618 & 0.327806 & -2.549098 \\
\hline $\mathrm{C}$ & -0.944124 & -2.286627 & 0.022228 \\
\hline $\mathrm{C}$ & 0.141340 & -2.435415 & -0.868013 \\
\hline $\mathrm{H}$ & -0.798171 & -2.515781 & 1.079142 \\
\hline $\mathrm{H}$ & -1.963912 & -2.412632 & -0.348016 \\
\hline $\mathrm{H}$ & -0.077271 & -2.515267 & -1.938744 \\
\hline $\mathrm{C}$ & 1.486577 & -2.202380 & -0.500943 \\
\hline $\mathrm{H}$ & 1.805282 & -2.383500 & 0.531015 \\
\hline $\mathrm{H}$ & 2.267107 & -2.304744 & -1.260071 \\
\hline $\mathrm{H}$ & 2.130456 & 0.563325 & -0.432362 \\
\hline $\mathrm{H}$ & 2.184427 & -0.234944 & -0.522641 \\
\hline $\mathrm{H}$ & -1.525757 & 1.986894 & 1.812976 \\
\hline $\mathrm{H}$ & 1.127756 & 2.576071 & 1.611104 \\
\hline $\mathrm{H}$ & 2.529713 & 0.332092 & 2.241085 \\
\hline $\mathrm{H}$ & 0.757176 & -1.641395 & 2.795073 \\
\hline $\mathrm{H}$ & -1.758405 & -0.618015 & 2.528779 \\
\hline $\mathrm{H}$ & 1.320829 & 2.107882 & -2.214323 \\
\hline $\mathrm{H}$ & -0.667991 & 3.038099 & -0.624017 \\
\hline $\mathrm{H}$ & -2.722433 & 1.245833 & -0.704145 \\
\hline $\mathrm{H}$ & -2.007738 & -0.769456 & -2.367967 \\
\hline & 51483 & -0.26424 & -3.2746 \\
\hline
\end{tabular}

Structure ISß2

$\begin{array}{llll}\mathrm{Zr} & -0.010324 & 0.075319 & 0.004629\end{array}$ 


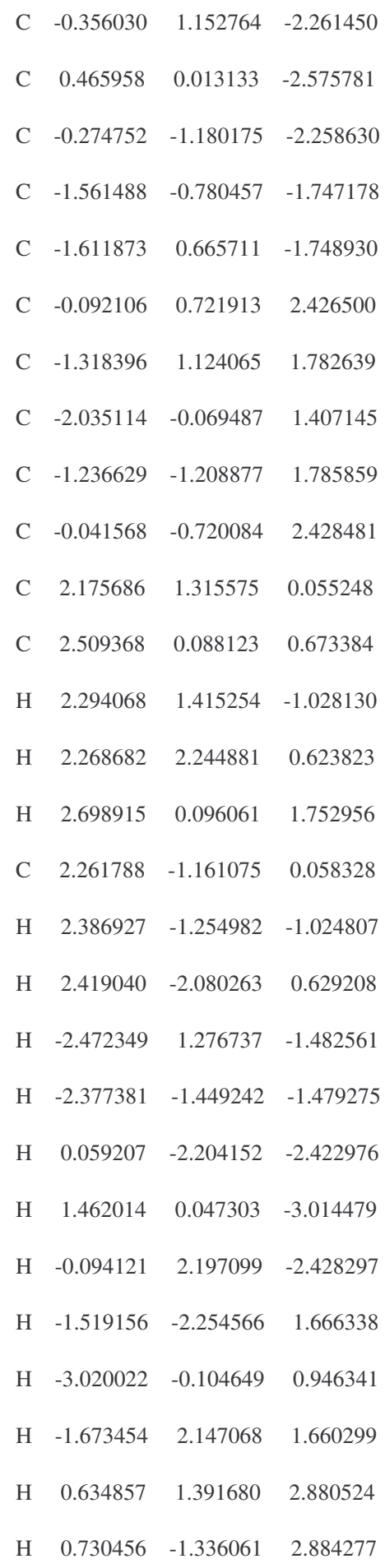

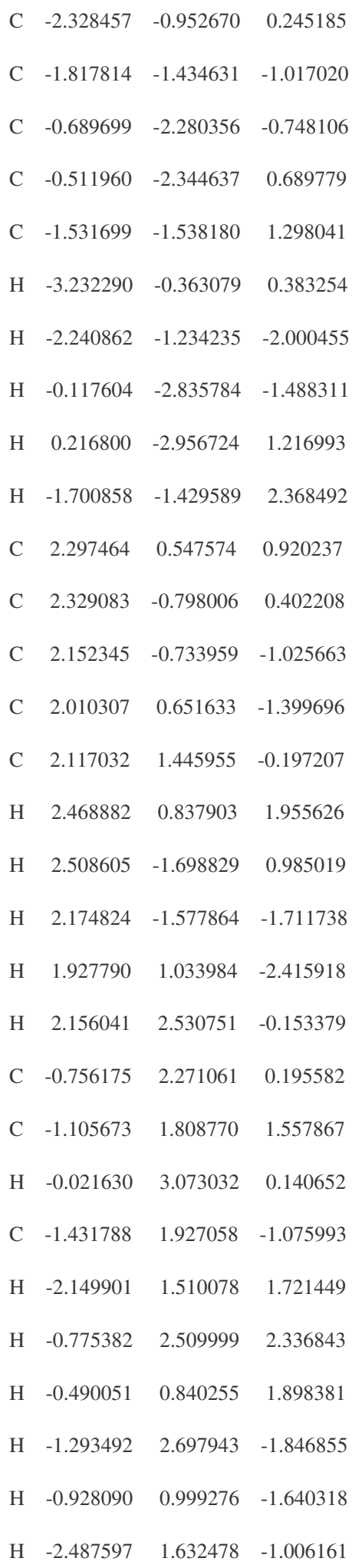




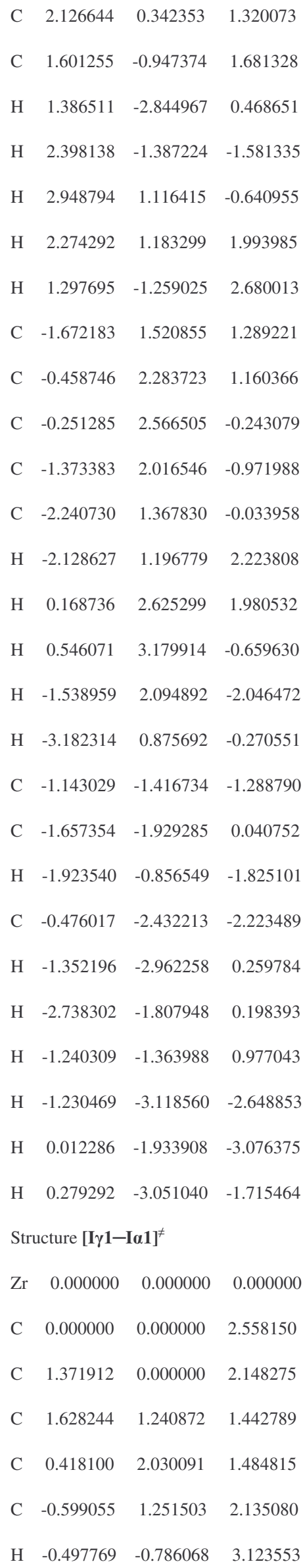

$\begin{array}{llll}\text { C } & 0.417468 & 2.029598 & 1.479954\end{array}$

$\begin{array}{llll}\text { C } & -0.597413 & 1.252757 & 2.135372\end{array}$

H $\quad-0.498905 \quad-0.788796 \quad 3.115152$

$\begin{array}{llll}\text { H } & 2.100463 & -0.785014 & 2.338578\end{array}$

H $2.593828 \quad 1.574425 \quad 1.060938$

$\begin{array}{llll}\text { H } & 0.302383 & 3.040114 & 1.093305\end{array}$

$\begin{array}{llll}\mathrm{H} & -1.614092 & 1.581729 & 2.352001\end{array}$

$\begin{array}{llll}\text { C } & -1.413763 & 0.795384 & -1.956656\end{array}$

$\begin{array}{llll}\text { C } & -0.453238 & 1.832175 & -1.694785\end{array}$

$\begin{array}{llll}\text { C } & 0.864499 & 1.298855 & -1.962792\end{array}$

$\begin{array}{llll}\text { C } & 0.701559 & -0.054046 & -2.452937\end{array}$

C $\quad-0.696628 \quad-0.369371 \quad-2.436063$

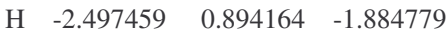

$\begin{array}{llll}\mathrm{H} & -0.679684 & 2.847181 & -1.374334\end{array}$

$\begin{array}{llll}\mathrm{H} & 1.798877 & 1.855797 & -1.920770\end{array}$

H $\quad 1.502007 \quad-0.715170 \quad-2.782648$

H $\quad-1.144476 \quad-1.307017 \quad-2.760788$

$\begin{array}{llll}\text { C } & 0.545477 & -2.161709 & -0.026892\end{array}$

$\begin{array}{llll}\text { C } & 0.199680 & -3.133781 & 1.121258\end{array}$

H $\quad 0.237403 \quad-2.609104 \quad-0.989054$

H $\quad 1.657754 \quad-2.046570 \quad-0.113152$

$\begin{array}{llll}\mathrm{H} & 0.658610 & -2.800837 & 2.067066\end{array}$

C $\quad 0.640750 \quad-4.584790 \quad 0.830843$

H $\quad 0.160637 \quad-4.973192 \quad-0.081702$

H $\quad 1.732167 \quad-4.645214 \quad 0.690922$

H $\quad 0.367074 \quad-5.250266 \quad 1.665240$

Н $\quad-0.894701 \quad-3.129309 \quad 1.291352$

Structure $[\mathbf{I} \boldsymbol{\gamma} \mathbf{1}-\mathbf{I} \boldsymbol{\beta} \mathbf{1}]^{\neq}$

$\begin{array}{lllll}\mathrm{Zr} & 0.017738 & 0.021391 & -0.120825\end{array}$

$\begin{array}{llll}\text { C } & 2.399816 & 0.832518 & 0.506787\end{array}$

C $\quad \begin{array}{llll}2.202623 & -0.440971 & 1.139780\end{array}$

$\begin{array}{llll}\text { C } & 2.075754 & -1.437874 & 0.102221\end{array}$

C $\quad 2.159280 \quad-0.773291 \quad-1.171951$

$\begin{array}{lllll}\text { C } & 2.355828 & 0.636317 & -0.920677\end{array}$

$\begin{array}{llll}\mathrm{H} & 2.563494 & 1.778722 & 1.018077\end{array}$ 


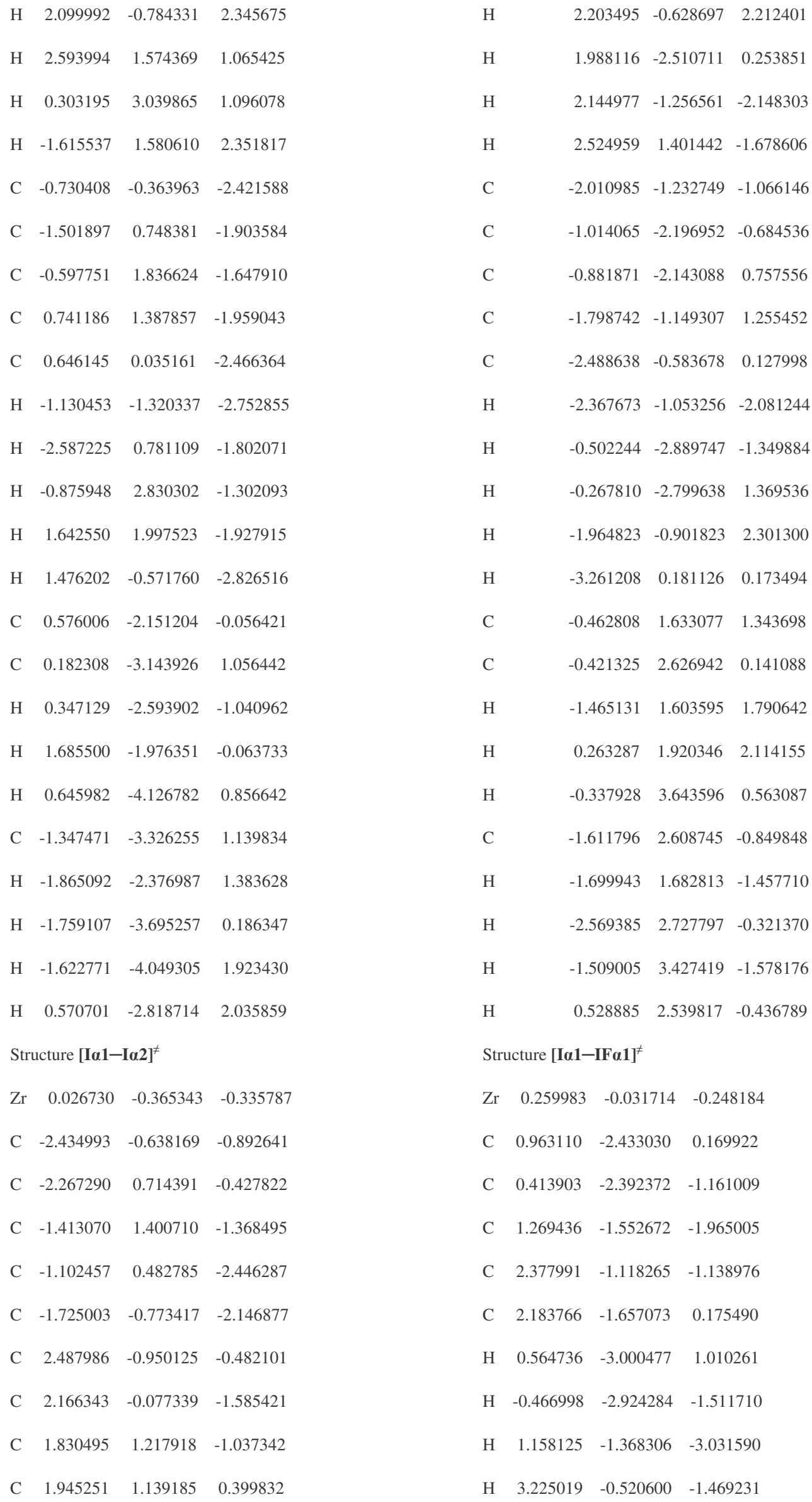




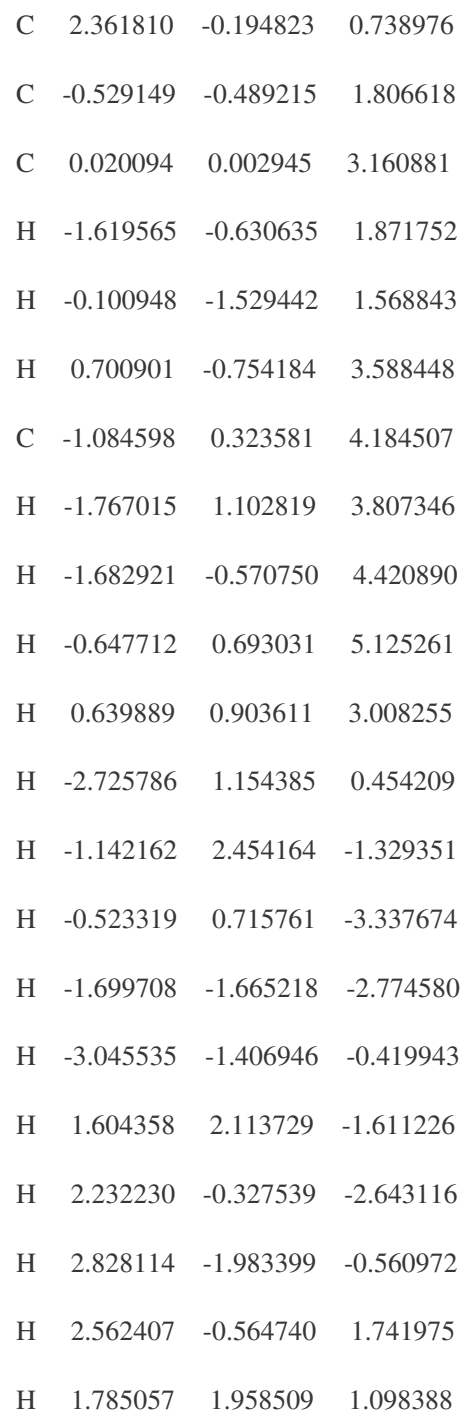

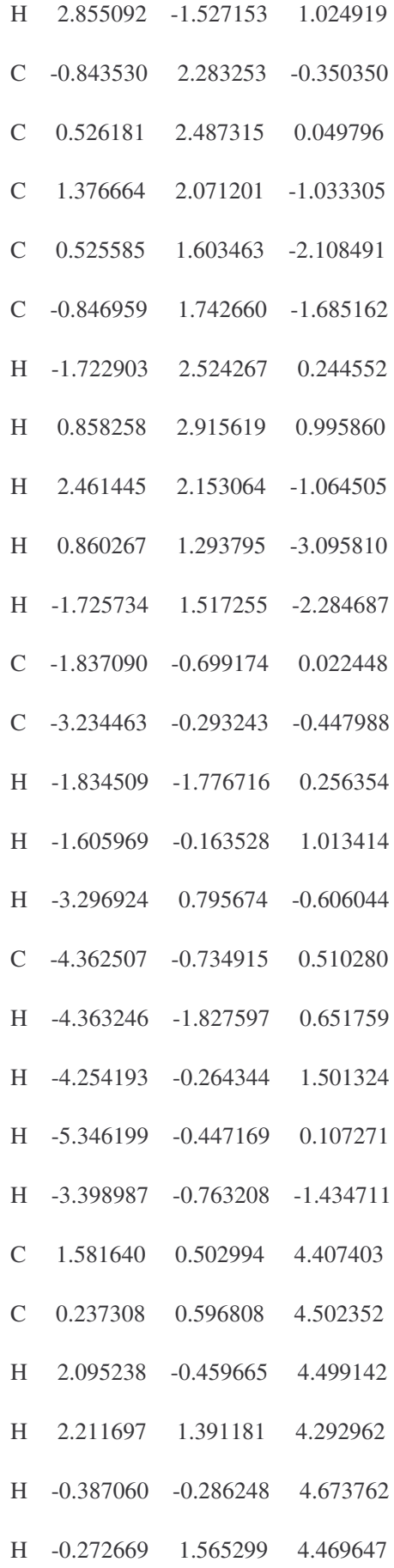




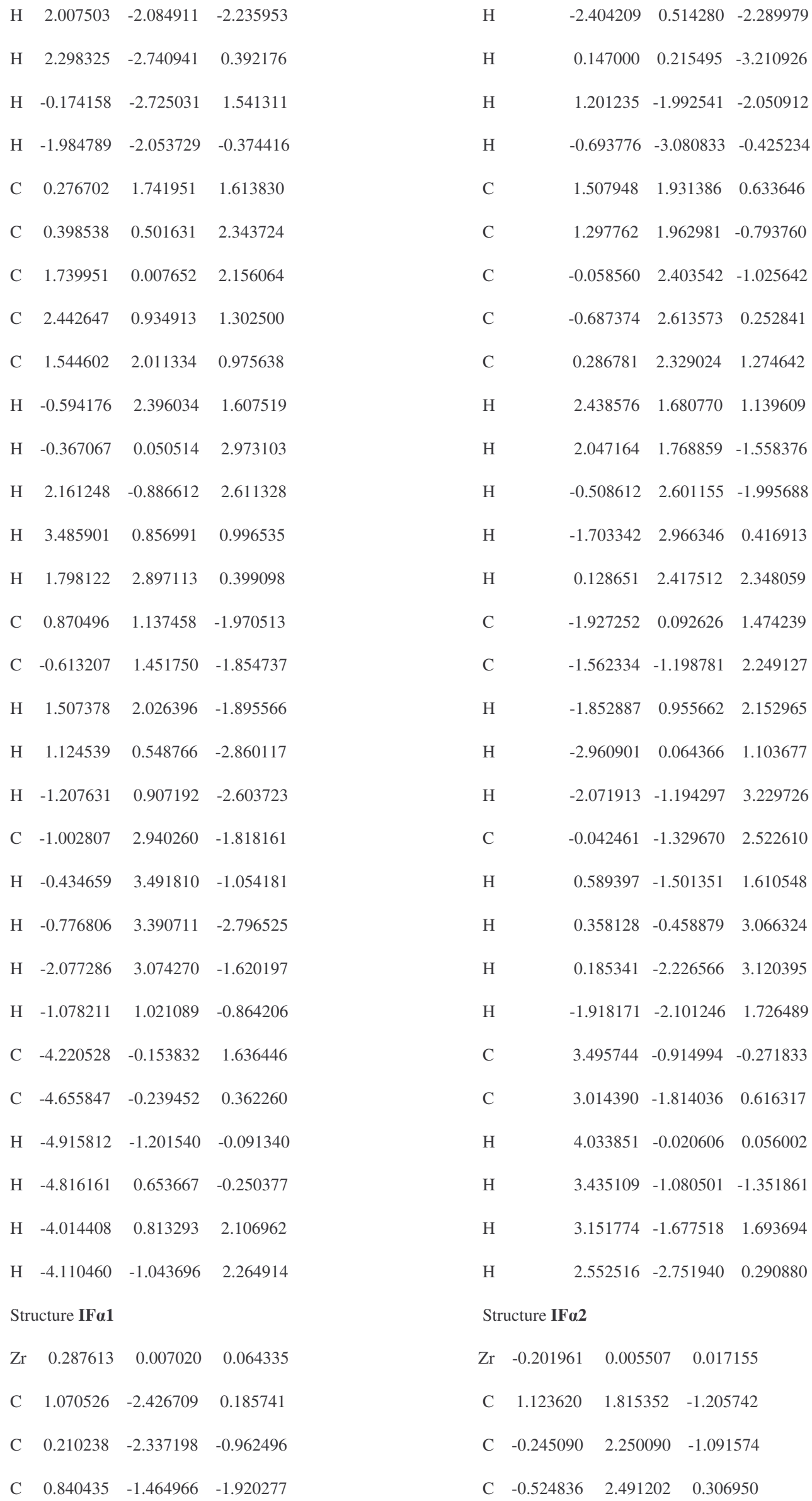

$\mathrm{H}$

$\mathrm{H}$

C

C

C

C

C

$\mathrm{H}$

$\mathrm{H}$

$\mathrm{H}$

$\mathrm{H}$

$\mathrm{H}$

C

C

$\mathrm{H}$

$\mathrm{H}$

$\mathrm{H}$

C

$\mathrm{H}$

$\mathrm{H}$

$\mathrm{H}$

$\mathrm{H}$

C

$\begin{array}{llll}\text { C } & 3.014390 & -1.814036 & 0.616317\end{array}$

H $\quad 4.033851 \quad-0.020606 \quad 0.056002$

$\begin{array}{llll}\mathrm{H} & 3.435109 & -1.080501 & -1.351861\end{array}$

$\begin{array}{llll}\mathrm{H} & 3.151774 & -1.677518 & 1.693694\end{array}$

$\mathrm{H}$ Structure IF $\mathbf{2}$

$\begin{array}{llll}\text { Zr } & -0.201961 & 0.005507 & 0.017155\end{array}$

C $\quad 1.123620 \quad 1.815352 \quad-1.205742$

$\begin{array}{llll}\text { C } & -0.245090 & 2.250090 & -1.091574\end{array}$

$\begin{array}{llll}\text { C } & -0.524836 & 2.491202 & 0.306950\end{array}$ 


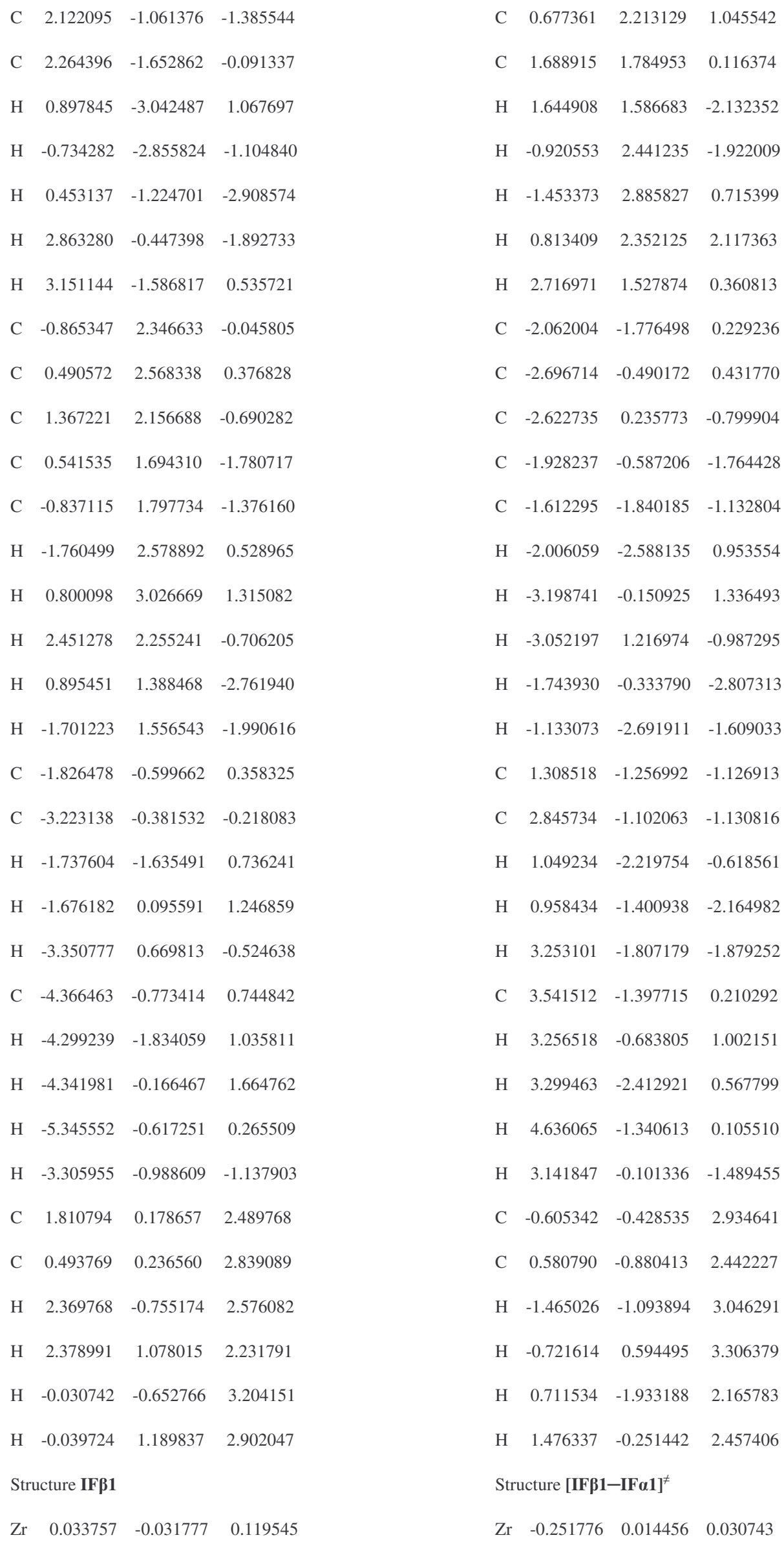




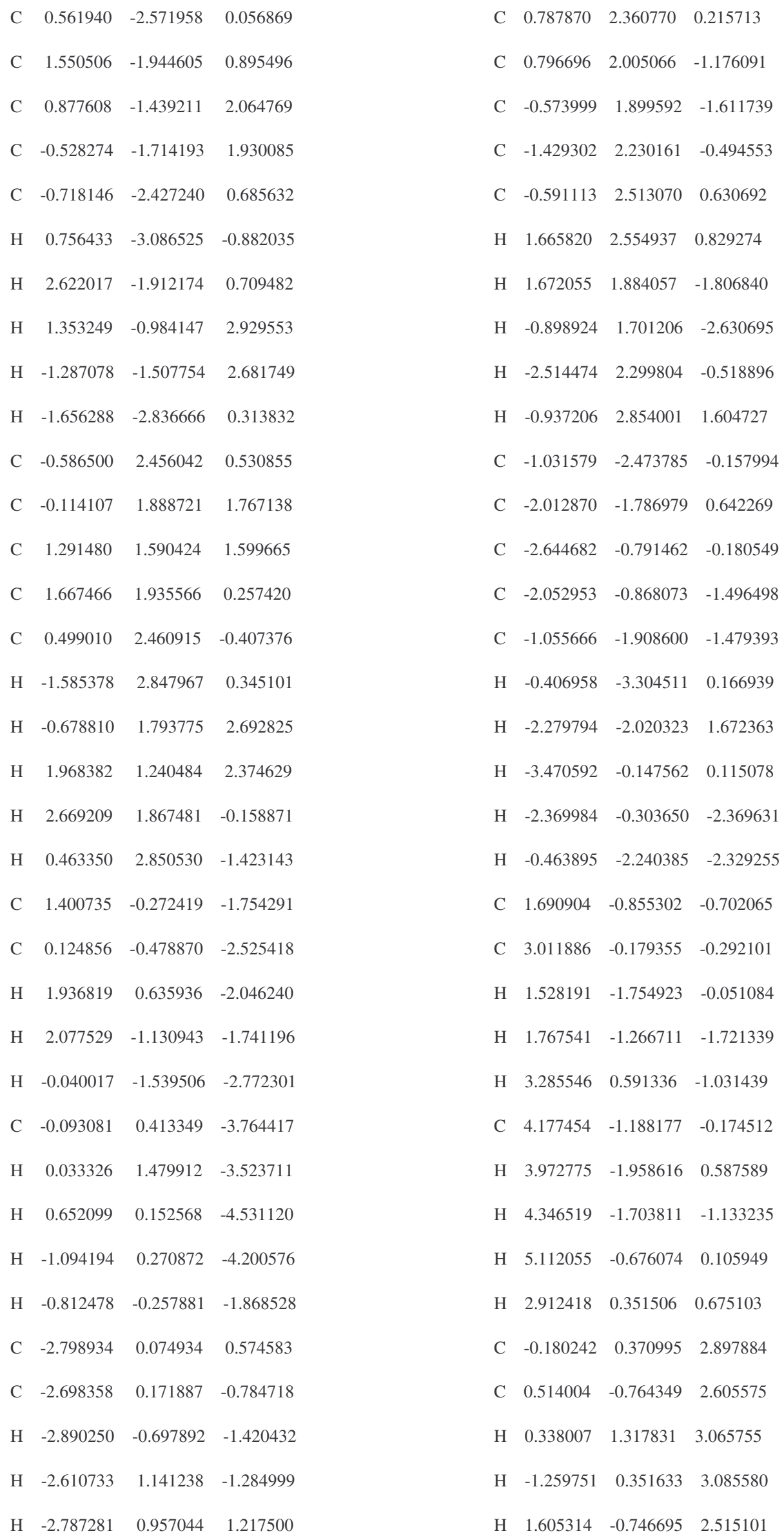




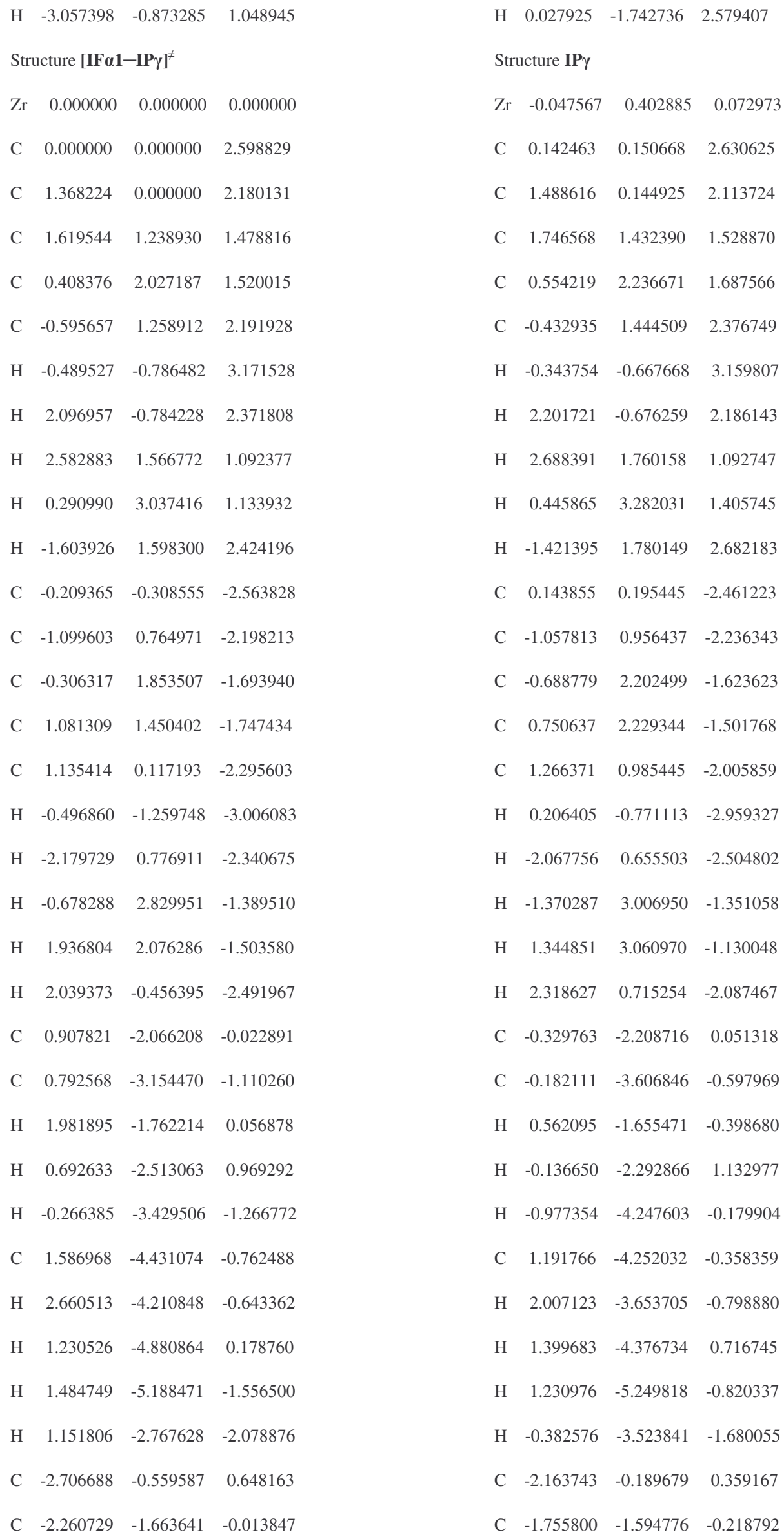




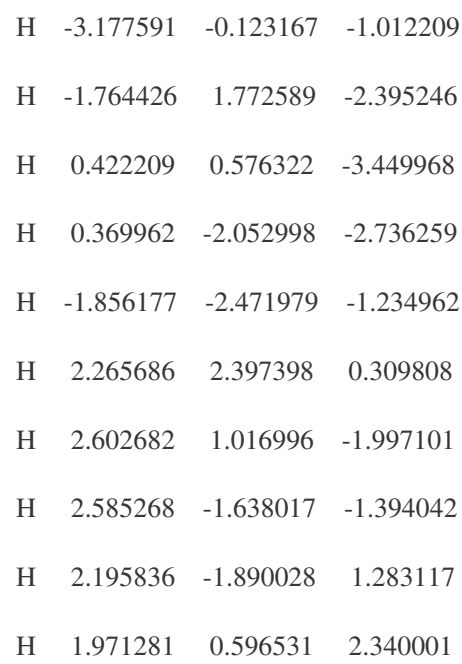

$\mathrm{H}$

$\mathrm{H}$

C

$\mathrm{H}$

$\mathrm{H}$

$\mathrm{H}$

C

$\mathrm{H}$

$\mathrm{H}$

$\mathrm{H}$

C

$\mathrm{H}$

$\mathrm{H}$

$\mathrm{H}$

C

$\mathrm{H}$

$\mathrm{H}$

$\mathrm{H}$

C

$\mathrm{H}$

$\mathrm{H}$

$\mathrm{H}$

C

$\mathrm{H}$

$\mathrm{H}$

$\mathrm{H}$

C

$\mathrm{H}$

$\mathrm{H}$

$\mathrm{H}$

C

$\begin{array}{llll}\mathrm{H} & -2.359620 & 2.280394 & 2.106403\end{array}$

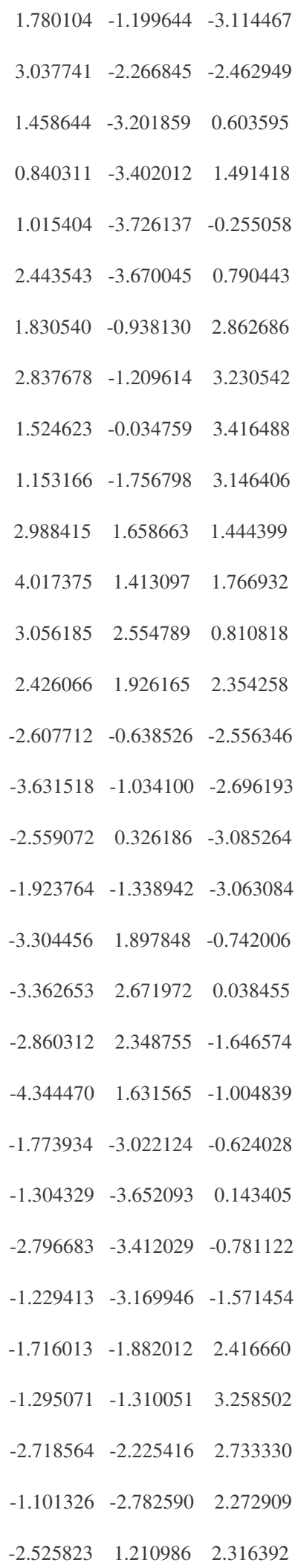




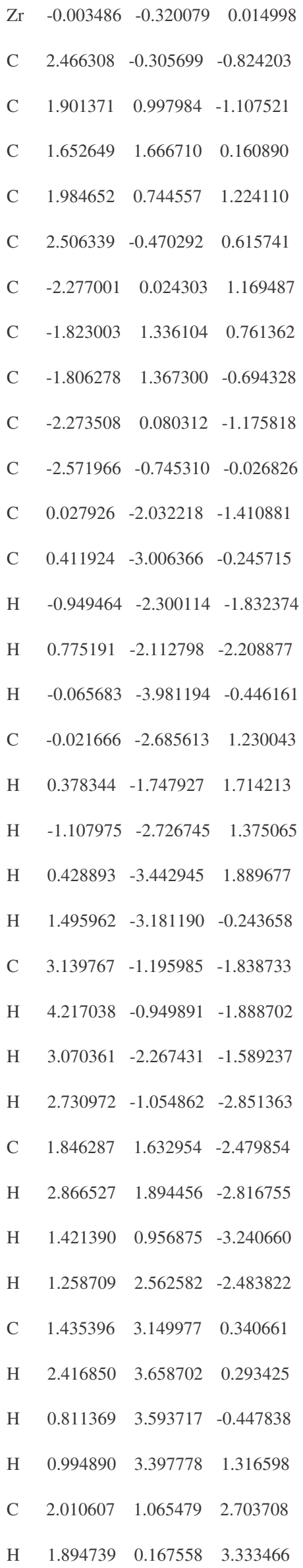

\begin{tabular}{|c|c|c|c|}
\hline $\mathrm{Zr}$ & 0.152177 & -0.137176 & -0.014812 \\
\hline $\mathrm{C}$ & -0.299981 & 0.840277 & -2.350252 \\
\hline $\mathrm{C}$ & -0.356266 & -0.588791 & -2.512670 \\
\hline $\mathrm{C}$ & -1.476592 & -1.087531 & -1.715151 \\
\hline $\mathrm{C}$ & -2.163747 & 0.051909 & -1.146806 \\
\hline $\mathrm{C}$ & -1.398103 & 1.243550 & -1.472870 \\
\hline $\mathrm{C}$ & 0.276376 & 1.243566 & 2.110557 \\
\hline C & -1.030054 & 0.620338 & 2.173660 \\
\hline $\mathrm{C}$ & -0.837480 & -0.816979 & 2.243185 \\
\hline C & 0.596507 & -1.070333 & 2.320963 \\
\hline $\mathrm{C}$ & 1.284587 & 0.198661 & 2.253224 \\
\hline $\mathrm{C}$ & 2.196722 & -0.840684 & -0.566198 \\
\hline $\mathrm{C}$ & 3.241225 & -0.530537 & -1.649686 \\
\hline $\mathrm{H}$ & 2.736739 & -1.096086 & 0.359981 \\
\hline $\mathrm{H}$ & 1.620071 & -1.782769 & -0.843254 \\
\hline $\mathrm{H}$ & 3.901803 & -1.405932 & -1.798064 \\
\hline $\mathrm{C}$ & 4.119731 & 0.679121 & -1.272746 \\
\hline $\mathrm{H}$ & 3.516401 & 1.594507 & -1.149034 \\
\hline $\mathrm{H}$ & 4.662213 & 0.501522 & -0.330088 \\
\hline $\mathrm{H}$ & 4.870179 & 0.874484 & -2.054866 \\
\hline $\mathrm{H}$ & 2.761996 & -0.343282 & -2.621611 \\
\hline $\mathrm{C}$ & 0.411751 & -1.430070 & -3.506404 \\
\hline $\mathrm{H}$ & 1.163639 & -0.841718 & -4.050967 \\
\hline $\mathrm{H}$ & 0.924112 & -2.294386 & -3.050823 \\
\hline $\mathrm{H}$ & -0.290110 & -1.833986 & -4.258414 \\
\hline $\mathrm{C}$ & -1.982585 & -2.515526 & -1.731902 \\
\hline $\mathrm{H}$ & -2.650597 & -2.671064 & -2.599857 \\
\hline $\mathrm{H}$ & -1.164716 & -3.248890 & -1.824985 \\
\hline $\mathrm{H}$ & -2.565302 & -2.765716 & -0.830907 \\
\hline $\mathrm{C}$ & -3.552391 & 0.013980 & -0.557126 \\
\hline $\mathrm{H}$ & -4.285658 & -0.060634 & -1.382240 \\
\hline $\mathrm{H}$ & -3.721438 & -0.858271 & 0.093721 \\
\hline $\mathrm{H}$ & -3.797696 & 0.924060 & 0.006534 \\
\hline C & -1.831684 & 2.677678 & -1.247997 \\
\hline H & -0.979421 & 3.359329 & -1.090155 \\
\hline
\end{tabular}




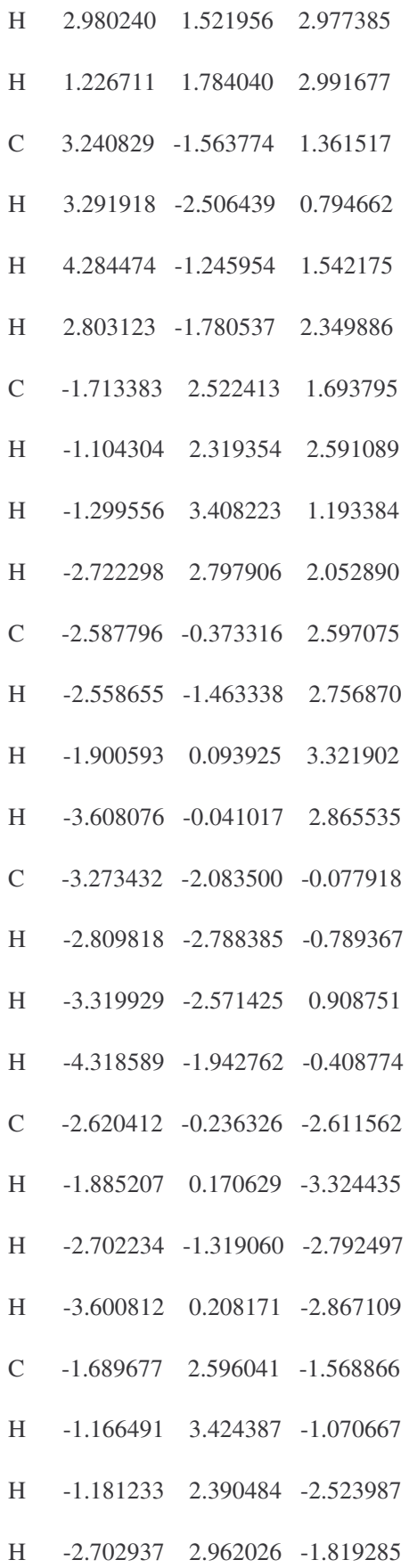

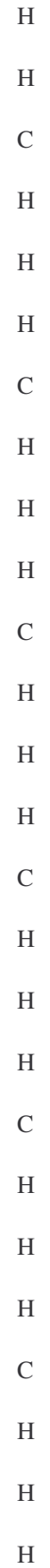




\begin{tabular}{|c|c|c|c|}
\hline C & -0.509742 & 0.634281 & 2.281505 \\
\hline $\mathrm{C}$ & -0.463837 & -0.820097 & 2.230534 \\
\hline C & 0.926424 & -1.212552 & 2.069769 \\
\hline $\mathrm{C}$ & 1.735266 & -0.015320 & 2.039704 \\
\hline $\mathrm{C}$ & 2.361999 & -0.152856 & -0.990374 \\
\hline $\mathrm{C}$ & 2.260287 & 1.364102 & -1.006150 \\
\hline $\mathrm{H}$ & 3.029763 & -0.473940 & -0.181391 \\
\hline $\mathrm{C}$ & 2.827947 & -0.817012 & -2.296354 \\
\hline $\mathrm{H}$ & 2.321109 & 1.787545 & -2.018255 \\
\hline $\mathrm{H}$ & 2.965095 & 1.885520 & -0.341006 \\
\hline $\mathrm{H}$ & 1.245910 & 1.791636 & -0.635188 \\
\hline $\mathrm{H}$ & 3.893716 & -0.593607 & -2.485508 \\
\hline $\mathrm{H}$ & 2.739235 & -1.913502 & -2.241993 \\
\hline $\mathrm{H}$ & 2.264276 & -0.481359 & -3.176607 \\
\hline $\mathrm{C}$ & 3.238497 & 0.035471 & 2.170094 \\
\hline $\mathrm{H}$ & 3.506638 & 0.226773 & 3.225691 \\
\hline $\mathrm{H}$ & 3.715915 & -0.916330 & 1.889135 \\
\hline $\mathrm{H}$ & 3.703047 & 0.840429 & 1.576681 \\
\hline $\mathrm{C}$ & 1.274044 & 2.567124 & 2.333477 \\
\hline $\mathrm{H}$ & 1.433451 & 2.767640 & 3.409520 \\
\hline $\mathrm{H}$ & 2.223380 & 2.797256 & 1.823972 \\
\hline $\mathrm{H}$ & 0.515557 & 3.285543 & 1.982744 \\
\hline $\mathrm{C}$ & -1.677785 & 1.483159 & 2.731191 \\
\hline $\mathrm{H}$ & -1.528619 & 1.774862 & 3.787441 \\
\hline $\mathrm{H}$ & -1.778874 & 2.418781 & 2.156413 \\
\hline $\mathrm{H}$ & -2.632452 & 0.943036 & 2.679425 \\
\hline $\mathrm{C}$ & -1.582339 & -1.763321 & 2.615239 \\
\hline $\mathrm{H}$ & -1.506359 & -2.740463 & 2.112584 \\
\hline $\mathrm{H}$ & -1.538022 & -1.959770 & 3.702724 \\
\hline $\mathrm{H}$ & -2.579179 & -1.348565 & 2.406667 \\
\hline C & 1.449662 & -2.632614 & 2.065185 \\
\hline $\mathrm{H}$ & 2.336052 & -2.753355 & 1.420303 \\
\hline $\mathrm{H}$ & 1.753552 & -2.929527 & 3.086309 \\
\hline $\mathrm{H}$ & 0.688230 & -3.358343 & 1.736607 \\
\hline C & 0.002946 & 1.361227 & -3.582957 \\
\hline
\end{tabular}

\begin{tabular}{|c|c|c|c|}
\hline $\mathrm{C}$ & 1.937785 & 0.881230 & -1.168101 \\
\hline $\mathrm{C}$ & 1.657357 & 1.621664 & 0.050200 \\
\hline $\mathrm{C}$ & 1.993990 & 0.772675 & 1.182019 \\
\hline $\mathrm{C}$ & 2.543321 & -0.462776 & 0.660341 \\
\hline $\mathrm{C}$ & 0.457984 & -2.632008 & 0.079970 \\
\hline $\mathrm{C}$ & -0.169526 & -2.576981 & -1.255932 \\
\hline $\mathrm{H}$ & 1.502709 & -2.936969 & 0.095382 \\
\hline $\mathrm{C}$ & -0.187640 & -2.512202 & 1.412757 \\
\hline $\mathrm{H}$ & -1.222959 & -2.874800 & -1.321164 \\
\hline $\mathrm{H}$ & 0.425401 & -3.106645 & -2.014248 \\
\hline $\mathrm{H}$ & -0.168318 & -1.480700 & -1.732824 \\
\hline $\mathrm{H}$ & 0.395218 & -3.015264 & 2.195922 \\
\hline $\mathrm{H}$ & -0.191836 & -1.398750 & 1.833685 \\
\hline $\mathrm{H}$ & -1.239538 & -2.812615 & 1.471071 \\
\hline $\mathrm{C}$ & 3.251475 & -1.510506 & 1.487489 \\
\hline $\mathrm{H}$ & 4.252453 & -1.138407 & 1.774142 \\
\hline $\mathrm{H}$ & 2.722054 & -1.747480 & 2.425227 \\
\hline $\mathrm{H}$ & 3.411982 & -2.449686 & 0.935282 \\
\hline $\mathrm{C}$ & 3.122905 & -1.393896 & -1.741716 \\
\hline $\mathrm{H}$ & 4.202804 & -1.182066 & -1.849595 \\
\hline $\mathrm{H}$ & 3.040219 & -2.436253 & -1.391503 \\
\hline $\mathrm{H}$ & 2.683102 & -1.337088 & -2.750111 \\
\hline $\mathrm{C}$ & 1.905172 & 1.441048 & -2.573846 \\
\hline $\mathrm{H}$ & 2.897410 & 1.850886 & -2.839966 \\
\hline $\mathrm{H}$ & 1.661159 & 0.675427 & -3.328792 \\
\hline $\mathrm{H}$ & 1.179590 & 2.261155 & -2.677285 \\
\hline $\mathrm{C}$ & 1.472505 & 3.119519 & 0.129089 \\
\hline $\mathrm{H}$ & 1.040995 & 3.442787 & 1.086256 \\
\hline $\mathrm{H}$ & 2.465758 & 3.600230 & 0.047027 \\
\hline $\mathrm{H}$ & 0.857784 & 3.526164 & -0.687006 \\
\hline $\mathrm{C}$ & 2.025813 & 1.201133 & 2.634016 \\
\hline $\mathrm{H}$ & 1.954462 & 0.343969 & 3.322806 \\
\hline $\mathrm{H}$ & 2.977416 & 1.718514 & 2.858252 \\
\hline $\mathrm{H}$ & 1.213460 & 1.902043 & 2.883430 \\
\hline $\mathrm{C}$ & .53714 & -0.029093 & 2.649535 \\
\hline
\end{tabular}




\begin{tabular}{|c|c|c|c|}
\hline $\mathrm{H}$ & 0.341405 & 2.364775 & -3.279115 \\
\hline $\mathrm{H}$ & 0.862430 & 0.826579 & -4.013691 \\
\hline $\mathrm{H}$ & -0.727100 & 1.508382 & -4.400849 \\
\hline $\mathrm{C}$ & -1.844726 & 2.704214 & -1.364928 \\
\hline $\mathrm{H}$ & -2.192375 & 2.998099 & -0.361664 \\
\hline $\mathrm{H}$ & -0.978413 & 3.336717 & -1.618686 \\
\hline $\mathrm{H}$ & -2.651548 & 2.964788 & -2.075035 \\
\hline $\mathrm{C}$ & -3.490193 & 0.346534 & 0.051942 \\
\hline $\mathrm{H}$ & -4.361036 & 0.302764 & -0.629665 \\
\hline $\mathrm{H}$ & -3.636874 & -0.445578 & 0.800545 \\
\hline $\mathrm{H}$ & -3.532041 & 1.321037 & 0.558146 \\
\hline $\mathrm{C}$ & -2.293320 & -2.473872 & -0.952569 \\
\hline $\mathrm{H}$ & -1.531353 & -3.270383 & -0.993123 \\
\hline $\mathrm{H}$ & -2.785393 & -2.522124 & 0.029808 \\
\hline $\mathrm{H}$ & -3.058160 & -2.728018 & -1.710302 \\
\hline $\mathrm{C}$ & -0.240369 & -1.881237 & -3.262668 \\
\hline $\mathrm{H}$ & 0.409299 & -1.456950 & -4.040438 \\
\hline $\mathrm{H}$ & 0.326605 & -2.676456 & -2.749024 \\
\hline $\mathrm{H}$ & -1.085832 & -2.375099 & -3.775812 \\
\hline
\end{tabular}

Structure IIA

$\mathrm{Zr} \quad \begin{array}{llll}0.347375 & -0.001385 & -0.020689\end{array}$

$\begin{array}{llll}\text { C } & 0.005534 & -1.141844 & 2.272285\end{array}$

$\begin{array}{llll}\text { C } & 0.879316 & -0.019087 & 2.549727\end{array}$

$\begin{array}{llll}\text { C } & 0.149189 & 1.200359 & 2.266255\end{array}$

$\begin{array}{llll}\text { C } & -1.191286 & 0.835034 & 1.849395\end{array}$

$\begin{array}{llll}\text { C } & -1.283723 & -0.618106 & 1.864317\end{array}$

$\begin{array}{llll}\text { C } & 0.353028 & -0.748194 & -2.498190\end{array}$

$\begin{array}{llll}\text { C } & -0.863040 & -1.255840 & -1.888259\end{array}$

C $\quad \begin{array}{llll} & -1.715283 & -0.122879 & -1.579111\end{array}$

$\begin{array}{lllll}\text { C } & & -0.984473 & 1.089826 & -1.906596\end{array}$

$\begin{array}{llll}\text { C } & 0.277410 & 0.701388 & -2.510218\end{array}$

$\begin{array}{llll}\text { C } & 2.545342 & -1.181338 & -0.017178\end{array}$

$\begin{array}{lllll}\text { C } & 2.789248 & 0.048617 & -0.665592\end{array}$

$\begin{array}{llll}\mathrm{H} & 2.661307 & -1.250998 & 1.065681\end{array}$

$\mathrm{H}$
$\mathrm{H}$

$\mathrm{H}$

$\mathrm{H}$

C

$\mathrm{H}$

$\mathrm{H}$

$\mathrm{H}$

C

$\mathrm{H}$

$\mathrm{H}$

$\mathrm{H}$

C

$\mathrm{H}$

$\mathrm{H}$

$\mathrm{H}$

C

$\mathrm{H}$

H

H

Structure IIH2

$\begin{array}{llll}\mathrm{Zr} & 0.243775 & -0.272689 & -0.105029 \\ \mathrm{C} & -0.458867 & -0.569228 & 2.394267 \\ \mathrm{C} & 0.947646 & -0.932619 & 2.350625 \\ \mathrm{C} & 1.703611 & 0.244810 & 1.991718 \\ \mathrm{C} & 0.771951 & 1.346082 & 1.813146 \\ \mathrm{C} & -0.564798 & 0.841638 & 2.089184 \\ \mathrm{C} & -1.158063 & -0.484677 & -2.337040 \\ \mathrm{C} & -1.904630 & 0.352801 & -1.420491 \\ \mathrm{C} & -1.162421 & 1.591862 & -1.248629 \\ \mathrm{C} & 0.088992 & 1.470481 & -1.963406 \\ \mathrm{C} & 0.095586 & 0.174060 & -2.635138 \\ \mathrm{C} & -0.512173 & -2.633537 & -0.033078 \\ \mathrm{C} & 0.567754 & -2.641804 & -0.944769 \\ \mathrm{H} & -0.324514 & -2.850232 & 1.017319 \\ \mathrm{H} & -1.509011 & -2.901417 & -0.389702 \\ & & & \end{array}$




\begin{tabular}{|c|c|c|c|c|c|c|c|}
\hline $\mathrm{H}$ & 2.996254 & 0.014438 & -1.739038 & $\mathrm{H}$ & 0.336511 & -2.763175 & -2.006549 \\
\hline $\mathrm{C}$ & 2.448676 & 1.303554 & -0.109130 & $\mathrm{C}$ & 1.877116 & -2.227601 & -0.622048 \\
\hline $\mathrm{H}$ & 2.565710 & 1.465136 & 0.964264 & $\mathrm{H}$ & 2.254208 & -2.348598 & 0.395853 \\
\hline $\mathrm{H}$ & 2.551366 & 2.197669 & -0.729592 & $\mathrm{H}$ & 2.634470 & -2.220342 & -1.411192 \\
\hline $\mathrm{C}$ & -3.199334 & -0.197717 & -1.315717 & $\mathrm{C}$ & -1.576066 & -1.415365 & 2.962611 \\
\hline $\mathrm{H}$ & -3.575183 & 0.637614 & -0.707284 & $\mathrm{H}$ & -2.541997 & -1.226338 & 2.467354 \\
\hline $\mathrm{H}$ & -3.728078 & -0.144821 & -2.286474 & $\mathrm{H}$ & -1.366210 & -2.494463 & 2.895598 \\
\hline $\mathrm{H}$ & -3.500076 & -1.141808 & -0.841361 & $\mathrm{H}$ & -1.711124 & -1.184428 & 4.036214 \\
\hline $\mathrm{C}$ & 1.355419 & -1.602157 & -3.240463 & $\mathrm{C}$ & -1.791707 & 1.671364 & 2.393323 \\
\hline $\mathrm{H}$ & 2.289294 & -1.064586 & -3.466299 & $\mathrm{H}$ & -1.704390 & 2.702688 & 2.027326 \\
\hline $\mathrm{H}$ & 1.614628 & -2.526512 & -2.699129 & $\mathrm{H}$ & -2.720358 & 1.235818 & 1.990465 \\
\hline $\mathrm{H}$ & 0.926073 & -1.910869 & -4.211586 & $\mathrm{H}$ & -1.920557 & 1.729154 & 3.490449 \\
\hline $\mathrm{C}$ & -1.258346 & -2.715953 & -1.826805 & $\mathrm{C}$ & 1.177292 & 2.800472 & 1.705767 \\
\hline $\mathrm{H}$ & -0.397063 & -3.375718 & -1.630643 & $\mathrm{H}$ & 2.044516 & 2.954018 & 1.042775 \\
\hline $\mathrm{H}$ & -2.019867 & -2.913015 & -1.056291 & $\mathrm{H}$ & 0.358504 & 3.439221 & 1.344806 \\
\hline $\mathrm{H}$ & -1.690196 & -3.033890 & -2.794220 & $\mathrm{H}$ & 1.465468 & 3.181776 & 2.703104 \\
\hline $\mathrm{C}$ & 1.194114 & 1.642246 & -3.256947 & $\mathrm{C}$ & 3.212250 & 0.363189 & 2.006138 \\
\hline $\mathrm{H}$ & 1.192469 & 2.656326 & -2.826981 & $\mathrm{H}$ & 3.567917 & 0.491508 & 3.045087 \\
\hline $\mathrm{H}$ & 2.237790 & 1.292167 & -3.297253 & $\mathrm{H}$ & 3.716539 & -0.530594 & 1.602867 \\
\hline $\mathrm{H}$ & 0.851018 & 1.736641 & -4.304068 & $\mathrm{H}$ & 3.566846 & 1.235677 & 1.435136 \\
\hline $\mathrm{C}$ & -1.544309 & 2.496417 & -1.888647 & $\mathrm{C}$ & 1.535310 & -2.226001 & 2.867941 \\
\hline $\mathrm{H}$ & -2.372028 & 2.604822 & -1.172255 & $\mathrm{H}$ & 1.419934 & -2.269242 & 3.966545 \\
\hline $\mathrm{H}$ & -0.780793 & 3.253602 & -1.643716 & $\mathrm{H}$ & 1.047209 & -3.132128 & 2.468603 \\
\hline $\mathrm{H}$ & -1.943151 & 2.757425 & -2.886620 & $\mathrm{H}$ & 2.614091 & -2.304168 & 2.659873 \\
\hline $\mathrm{C}$ & 0.607541 & 2.605443 & 2.590747 & $\mathrm{C}$ & -3.334262 & 0.103235 & -0.992814 \\
\hline $\mathrm{H}$ & 0.210594 & 2.909957 & 3.577250 & $\mathrm{H}$ & -4.035064 & 0.473285 & -1.764702 \\
\hline $\mathrm{H}$ & 1.704190 & 2.686800 & 2.650135 & $\mathrm{H}$ & -3.543735 & -0.969509 & -0.854130 \\
\hline $\mathrm{H}$ & 0.251839 & 3.349481 & 1.859039 & $\mathrm{H}$ & -3.589316 & 0.620631 & -0.055072 \\
\hline $\mathrm{C}$ & 2.220167 & -0.095257 & 3.242533 & $\mathrm{C}$ & -1.723655 & -1.689488 & -3.050866 \\
\hline $\mathrm{H}$ & 2.115774 & 0.260744 & 4.283802 & $\mathrm{H}$ & -0.974180 & -2.197612 & -3.676882 \\
\hline $\mathrm{H}$ & 2.600990 & -1.127019 & 3.298194 & $\mathrm{H}$ & -2.174154 & -2.433929 & -2.373754 \\
\hline $\mathrm{H}$ & 3.001396 & 0.529947 & 2.775517 & $\mathrm{H}$ & -2.530218 & -1.358664 & -3.730841 \\
\hline $\mathrm{C}$ & -2.342299 & 1.812969 & 1.761755 & $\mathrm{C}$ & -1.748503 & 2.894337 & -0.758882 \\
\hline $\mathrm{H}$ & -2.684391 & 2.068522 & 2.782080 & $\mathrm{H}$ & -1.006150 & 3.556078 & -0.288398 \\
\hline $\mathrm{H}$ & -2.057924 & 2.760470 & 1.276453 & $\mathrm{H}$ & -2.152552 & 3.443446 & -1.630758 \\
\hline
\end{tabular}




$\begin{array}{llll}\mathrm{H} & -3.211294 & 1.401230 & 1.229124 \\ \mathrm{C} & -2.554165 & -1.438204 & 1.820096 \\ \mathrm{H} & -2.453718 & -2.364651 & 1.230876 \\ \mathrm{H} & -2.823348 & -1.744111 & 2.847916 \\ \mathrm{H} & -3.404383 & -0.868445 & 1.422149 \\ \mathrm{C} & 0.301799 & -2.597527 & 2.562564 \\ \mathrm{H} & 1.352479 & -2.867542 & 2.368589 \\ \mathrm{H} & 0.103674 & -2.818828 & 3.628031 \\ \mathrm{H} & -0.333834 & -3.275507 & 1.970883\end{array}$

\section{Structure IIH1}

$\begin{array}{llll}\mathrm{Zr} & 0.041332 & 0.220952 & 0.014033\end{array}$

$\begin{array}{lllll}\text { C } & 2.347689 & -0.105833 & -1.053481\end{array}$

$\begin{array}{llll}\text { C } & 2.587125 & 0.037882 & 0.367430\end{array}$

$\begin{array}{llll}\text { C } & 1.986181 & -1.098517 & 1.044156\end{array}$

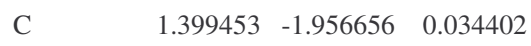

C $\quad \begin{array}{llll}1.596056 & -1.329396 & -1.264120\end{array}$

$\begin{array}{lllll}\text { C } & -2.366710 & 1.045897 & -0.284570\end{array}$

C $\quad \begin{array}{llll}-2.122264 & 0.036021 & -1.308749\end{array}$

$\begin{array}{llll}\text { C } & -1.993281 & -1.243668 & -0.649414\end{array}$

$\begin{array}{llll}\text { C } & -2.072037 & -1.019156 & 0.785721\end{array}$

$\begin{array}{llll}\text { C } & -2.336182 & 0.390518 & 1.007065\end{array}$

$\begin{array}{llll}\text { C } & 0.617652 & 2.691940 & -0.432803\end{array}$

$\begin{array}{llll}\text { C } & 0.161000 & 3.086484 & 0.795751\end{array}$

$\begin{array}{llll}\mathrm{H} & 1.662868 & 2.822364 & -0.717718\end{array}$

$\mathrm{H} \quad-0.100984 \quad 2.491541 \quad-1.245901$

$\begin{array}{llll}\mathrm{H} & -0.915465 & 3.040639 & 0.987052\end{array}$

$\begin{array}{llll}\text { C } & 0.992260 & 3.677481 & 1.891920\end{array}$

$\begin{array}{llll}\mathrm{H} & 0.651855 & 4.714662 & 2.065524\end{array}$

$\begin{array}{llll}\mathrm{H} & 0.834363 & 3.135773 & 2.838225\end{array}$

$\begin{array}{llll}\text { C } & 2.930150 & 0.742553 & -2.163650\end{array}$

$\begin{array}{llll}\mathrm{H} & 2.201688 & 0.973068 & -2.960582\end{array}$

$\begin{array}{lllll}\mathrm{H} & 3.346358 & 1.692741 & -1.794451\end{array}$

$\begin{array}{llll}\mathrm{H} & 3.763118 & 0.199701 & -2.647468\end{array}$
$\mathrm{H}$

C

$\mathrm{H}$

$\mathrm{H}$

$\mathrm{H}$

C

H

$\mathrm{H}$

$\mathrm{H}$

$\mathrm{H}$ Structure $[\mathbf{I I} \boldsymbol{\beta} 1-\mathbf{I I} \boldsymbol{\alpha} 2]^{\neq}$

$\begin{array}{llll}\mathrm{Zr} & 0.132395 & -0.095257 & -0.027485\end{array}$

$\begin{array}{llll}\text { C } & -0.487576 & 0.902394 & -2.313989\end{array}$

$\begin{array}{lllll}\text { C } & & -0.394218 & -0.518493 & -2.519747\end{array}$

$\begin{array}{llll}\text { C } & -1.436561 & -1.157891 & -1.715077\end{array}$

$\begin{array}{llll}\text { C } & -2.223637 & -0.115597 & -1.092818\end{array}$

$\begin{array}{lllll}\text { C } & & -1.595851 & 1.159713 & -1.396052\end{array}$

$\begin{array}{llll}\text { C } & 0.146806 & 1.257151 & 2.117995\end{array}$

$\begin{array}{llll}\text { C } & -1.059276 & 0.460033 & 2.216254\end{array}$

$\begin{array}{llll}\text { C } & -0.671087 & -0.937904 & 2.247155\end{array}$

$\begin{array}{llll}\text { C } & 0.786393 & -0.994571 & 2.271872\end{array}$

$\begin{array}{llll}\text { C } & 1.292654 & 0.356682 & 2.204516\end{array}$

$\begin{array}{llll}\text { C } & 2.220101 & -0.553184 & -0.668076\end{array}$

C $\quad \begin{array}{llll}3.179675 & -0.030109 & -1.764845\end{array}$

$\begin{array}{llll}\mathrm{H} & 2.808295 & -0.796334 & 0.230278\end{array}$

$\mathrm{H} \quad \begin{array}{llll}1.756462 & -1.539352 & -0.985585\end{array}$

H $\quad 3.221612 \quad-0.738738 \quad-2.608986$

$\begin{array}{llll}\text { C } & 4.612592 & 0.204383 & -1.250634\end{array}$

$\begin{array}{lllll}\mathrm{H} & 4.629687 & 0.932834 & -0.423382\end{array}$

H $\quad \begin{array}{llll}5.062882 & -0.733323 & -0.886121\end{array}$

$\begin{array}{llll}\mathrm{H} & 5.258954 & 0.597659 & -2.051623\end{array}$

$\begin{array}{llll}\mathrm{H} & 2.801384 & 0.915801 & -2.189864\end{array}$

C $\quad \begin{array}{llll}0.448885 & -1.241518 & -3.543154\end{array}$

$\mathrm{H} \quad \begin{array}{llll}1.265159 & -0.615823 & -3.929282\end{array}$ 


\begin{tabular}{|c|c|c|c|}
\hline $\mathrm{C}$ & 1.363706 & -1.961570 & -2.619424 \\
\hline $\mathrm{H}$ & 0.620645 & -2.770762 & -2.581618 \\
\hline $\mathrm{H}$ & 1.036187 & -1.232268 & -3.379624 \\
\hline $\mathrm{H}$ & 2.305494 & -2.407070 & -2.990875 \\
\hline $\mathrm{C}$ & 0.988939 & -3.390872 & 0.277482 \\
\hline $\mathrm{H}$ & 0.393133 & -3.517372 & 1.194288 \\
\hline $\mathrm{H}$ & 0.429100 & -3.818971 & -0.565419 \\
\hline $\mathrm{H}$ & 1.898980 & -4.006960 & 0.402638 \\
\hline $\mathrm{C}$ & 2.175480 & -1.456484 & 2.500558 \\
\hline $\mathrm{H}$ & 3.102179 & -2.047186 & 2.629541 \\
\hline $\mathrm{H}$ & 2.258530 & -0.562810 & 3.137366 \\
\hline $\mathrm{H}$ & 1.344018 & -2.065451 & 2.888058 \\
\hline C & 3.481092 & 1.053261 & 1.037492 \\
\hline $\mathrm{H}$ & 4.480981 & 0.611873 & 1.206870 \\
\hline $\mathrm{H}$ & 3.633060 & 1.957150 & 0.426653 \\
\hline $\mathrm{H}$ & 3.094150 & 1.359766 & 2.021959 \\
\hline $\mathrm{C}$ & -2.240438 & 0.257608 & -2.801879 \\
\hline $\mathrm{H}$ & -3.285840 & 0.101899 & -3.128774 \\
\hline $\mathrm{H}$ & -1.966128 & 1.283741 & -3.098975 \\
\hline $\mathrm{H}$ & -1.617395 & -0.442588 & -3.381491 \\
\hline C & -2.899602 & 2.436906 & -0.566291 \\
\hline $\mathrm{H}$ & -2.997439 & 3.039869 & 0.350102 \\
\hline $\mathrm{H}$ & -2.300249 & 3.013600 & -1.291846 \\
\hline $\mathrm{H}$ & -3.914865 & 2.355559 & -0.995292 \\
\hline $\mathrm{C}$ & -2.086211 & -2.579996 & -1.346368 \\
\hline $\mathrm{H}$ & -1.684658 & -3.405386 & -0.742939 \\
\hline $\mathrm{H}$ & -3.152841 & -2.809170 & -1.531050 \\
\hline $\mathrm{H}$ & -1.587234 & -2.586735 & -2.327158 \\
\hline C & -2.148205 & -2.077574 & 1.863221 \\
\hline $\mathrm{H}$ & -1.552545 & -1.812336 & 2.752280 \\
\hline $\mathrm{H}$ & -3.194503 & -2.199525 & 2.199397 \\
\hline $\mathrm{H}$ & -1.809757 & -3.060337 & 1.504645 \\
\hline C & -2.736584 & 0.993930 & 2.333269 \\
\hline $\mathrm{H}$ & -2.236189 & 0.493388 & 3.175616 \\
\hline $\mathrm{H}$ & 5341 & 2.069008 & 2.40220 \\
\hline
\end{tabular}

\begin{tabular}{|c|c|c|c|}
\hline $\mathrm{H}$ & 0.892498 & -2.173874 & -3.154751 \\
\hline $\mathrm{H}$ & -0.184782 & -1.523959 & -4.403977 \\
\hline $\mathrm{C}$ & -1.789683 & -2.630653 & -1.760107 \\
\hline $\mathrm{H}$ & -2.452170 & -2.836034 & -2.621721 \\
\hline $\mathrm{H}$ & -0.901988 & -3.272978 & -1.883090 \\
\hline $\mathrm{H}$ & -2.327142 & -2.960573 & -0.856614 \\
\hline $\mathrm{C}$ & -3.582360 & -0.317425 & -0.467687 \\
\hline $\mathrm{H}$ & -4.326521 & -0.458722 & -1.274055 \\
\hline $\mathrm{H}$ & -3.635906 & -1.213804 & 0.170490 \\
\hline $\mathrm{H}$ & -3.909096 & 0.550736 & 0.120327 \\
\hline $\mathrm{C}$ & -2.171344 & 2.533489 & -1.120549 \\
\hline $\mathrm{H}$ & -1.389873 & 3.305776 & -1.031389 \\
\hline $\mathrm{H}$ & -2.829351 & 2.840061 & -1.954970 \\
\hline $\mathrm{H}$ & -2.781506 & 2.559676 & -0.205174 \\
\hline $\mathrm{C}$ & 0.298964 & 1.984628 & -3.018872 \\
\hline $\mathrm{H}$ & 0.760536 & 2.709530 & -2.324214 \\
\hline $\mathrm{H}$ & 1.097209 & 1.570245 & -3.650414 \\
\hline $\mathrm{H}$ & -0.373051 & 2.565496 & -3.676659 \\
\hline $\mathrm{C}$ & 0.222440 & 2.768676 & 2.181851 \\
\hline $\mathrm{H}$ & 1.086603 & 3.172406 & 1.627579 \\
\hline $\mathrm{H}$ & -0.688350 & 3.250113 & 1.792761 \\
\hline $\mathrm{H}$ & 0.339147 & 3.097947 & 3.231202 \\
\hline $\mathrm{C}$ & 2.721576 & 0.794633 & 2.421381 \\
\hline $\mathrm{H}$ & 2.875057 & 1.038235 & 3.489283 \\
\hline $\mathrm{H}$ & 3.447431 & 0.007912 & 2.164650 \\
\hline $\mathrm{H}$ & 2.981038 & 1.695904 & 1.843009 \\
\hline $\mathrm{C}$ & -2.434841 & 1.007130 & 2.510764 \\
\hline $\mathrm{H}$ & -2.660955 & 1.930951 & 1.955696 \\
\hline $\mathrm{H}$ & -3.231990 & 0.277325 & 2.313677 \\
\hline $\mathrm{H}$ & -2.492315 & 1.262699 & 3.585631 \\
\hline $\mathrm{C}$ & -1.588316 & -2.115924 & 2.501926 \\
\hline $\mathrm{H}$ & -2.616962 & -1.927424 & 2.158693 \\
\hline $\mathrm{H}$ & -1.234036 & -3.041920 & 2.019325 \\
\hline $\mathrm{H}$ & -1.643274 & -2.323657 & 3.586578 \\
\hline$C$ & 1.607940 & -2.245170 & 2.492638 \\
\hline
\end{tabular}




\begin{tabular}{|c|c|c|c|c|c|c|c|}
\hline $\mathrm{H}$ & -3.827928 & 0.887569 & 2.480665 & $\mathrm{H}$ & 1.119289 & -3.148138 & 2.091332 \\
\hline $\mathrm{H}$ & 0.123616 & 0.580295 & 1.819008 & $\mathrm{H}$ & 2.612280 & -2.176653 & 2.045578 \\
\hline $\mathrm{H}$ & 2.064140 & 3.698077 & 1.651462 & $\mathrm{H}$ & 1.748303 & -2.412561 & 3.576724 \\
\hline \multicolumn{4}{|c|}{ Structure $[\mathbf{I I} \gamma \mathbf{1}-\mathbf{I I} \alpha 2]^{\neq}$} & \multicolumn{4}{|c|}{ Structure $[\mathbf{I I} \gamma \mathbf{1}-\mathbf{I I} \boldsymbol{\beta} \mathbf{1}]^{\neq}$} \\
\hline $\mathrm{Zr}$ & 0.003138 & 0.195088 & -0.092589 & $\mathrm{Zr}$ & -0.011150 & 0.278893 & -0.075644 \\
\hline $\mathrm{C}$ & -2.365439 & 0.182118 & 0.882019 & $\mathrm{C}$ & -2.626358 & 0.424267 & 0.165708 \\
\hline $\mathrm{C}$ & -2.529360 & 0.270014 & -0.552085 & $\mathrm{C}$ & -2.299371 & -0.191193 & -1.106342 \\
\hline $\mathrm{C}$ & -2.007972 & -0.963661 & -1.128766 & $\mathrm{C}$ & -1.668802 & -1.471907 & -0.841845 \\
\hline $\mathrm{C}$ & -1.606988 & -1.838773 & -0.043800 & $\mathrm{C}$ & -1.578506 & -1.629120 & 0.602847 \\
\hline $\mathrm{C}$ & -1.778829 & -1.113107 & 1.200624 & $\mathrm{C}$ & -2.149104 & -0.443554 & 1.218660 \\
\hline $\mathrm{C}$ & 2.506471 & 0.796471 & 0.026501 & $\mathrm{C}$ & 2.425126 & 0.569846 & -0.779851 \\
\hline $\mathrm{C}$ & 2.181960 & -0.004243 & 1.203510 & $\mathrm{C}$ & 2.465210 & 0.612721 & 0.669675 \\
\hline $\mathrm{C}$ & 1.888340 & -1.351333 & 0.764810 & $\mathrm{C}$ & 2.046258 & -0.683947 & 1.162035 \\
\hline $\mathrm{C}$ & 1.931367 & -1.367133 & -0.689862 & $\mathrm{C}$ & 1.835396 & -1.555772 & 0.015209 \\
\hline $\mathrm{C}$ & 2.340630 & -0.040731 & -1.135496 & $\mathrm{C}$ & 2.026802 & -0.770204 & -1.183092 \\
\hline $\mathrm{C}$ & -0.124857 & 2.202248 & -1.134397 & $\mathrm{C}$ & -0.150590 & 2.076711 & 1.271651 \\
\hline $\mathrm{C}$ & -0.786314 & 3.422241 & -0.446598 & $\mathrm{C}$ & 0.215707 & 3.126757 & 0.179974 \\
\hline $\mathrm{H}$ & 0.882984 & 2.499115 & -1.470738 & $\mathrm{H}$ & -1.148917 & 2.287136 & 1.681107 \\
\hline $\mathrm{H}$ & -0.686476 & 1.956311 & -2.063476 & $\mathrm{H}$ & 0.566451 & 2.138812 & 2.102231 \\
\hline $\mathrm{H}$ & -0.750093 & 4.295171 & -1.124599 & $\mathrm{H}$ & 0.517843 & 4.062200 & 0.685185 \\
\hline $\mathrm{C}$ & -0.119645 & 3.817970 & 0.885381 & $\mathrm{C}$ & -0.878970 & 3.474313 & -0.851519 \\
\hline $\mathrm{H}$ & -0.136201 & 2.990845 & 1.622133 & $\mathrm{H}$ & -1.211974 & 2.605847 & -1.452073 \\
\hline $\mathrm{H}$ & 0.930904 & 4.111285 & 0.737000 & $\mathrm{H}$ & -1.772893 & 3.884663 & -0.360350 \\
\hline $\mathrm{H}$ & -0.638821 & 4.669798 & 1.353265 & $\mathrm{H}$ & -0.508211 & 4.224051 & -1.568198 \\
\hline $\mathrm{H}$ & -1.855226 & 3.229401 & -0.269298 & $\mathrm{H}$ & 1.127540 & 2.824888 & -0.372456 \\
\hline $\mathrm{C}$ & -2.872746 & 1.174444 & 1.905295 & $\mathrm{C}$ & -3.526713 & 1.616537 & 0.378381 \\
\hline $\mathrm{H}$ & -2.293330 & 1.147209 & 2.842847 & $\mathrm{H}$ & -3.194639 & 2.264479 & 1.205793 \\
\hline $\mathrm{H}$ & -2.868640 & 2.209721 & 1.532310 & $\mathrm{H}$ & -3.629234 & 2.237550 & -0.523497 \\
\hline $\mathrm{H}$ & -3.917583 & 0.929175 & 2.173173 & $\mathrm{H}$ & -4.539673 & 1.259526 & 0.642569 \\
\hline $\mathrm{C}$ & -1.664221 & -1.690084 & 2.596184 & $\mathrm{C}$ & -2.411409 & -0.261061 & 2.694750 \\
\hline $\mathrm{H}$ & -1.006696 & -2.570811 & 2.628562 & $\mathrm{H}$ & -1.704470 & -0.832401 & 3.316406 \\
\hline $\mathrm{H}$ & -1.290996 & -0.959541 & 3.333794 & $\mathrm{H}$ & -2.355424 & 0.795388 & 3.002015 \\
\hline $\mathrm{H}$ & -2.659835 & -2.016334 & 2.949635 & $\mathrm{H}$ & -3.427947 & -0.618098 & 2.946682 \\
\hline $\mathrm{C}$ & -1.346006 & -3.319138 & -0.181105 & $\mathrm{C}$ & -1.322074 & -2.919345 & 1.348939 \\
\hline $\mathrm{H}$ & -0.868199 & -3.580742 & -1.136819 & $\mathrm{H}$ & -0.848300 & -3.681576 & 0.716669 \\
\hline
\end{tabular}




\begin{tabular}{|c|c|c|c|c|c|c|c|c|}
\hline $\mathrm{H}$ & -0.736583 & -3.726211 & 0.638312 & $\mathrm{H}$ & \multicolumn{2}{|c|}{-0.704704} & -2.783588 & 2.251519 \\
\hline $\mathrm{H}$ & -2.314283 & -3.853800 & -0.152916 & $\mathrm{H}$ & \multicolumn{2}{|c|}{-2.289671} & -3.334853 & 1.686290 \\
\hline $\mathrm{C}$ & -2.113328 & -1.342408 & -2.590732 & $\mathrm{C}$ & & 1.459075 & -2.549023 & -1.883108 \\
\hline $\mathrm{H}$ & -3.116644 & -1.757841 & -2.802085 & $\mathrm{H}$ & & 2.416134 & -3.070518 & -2.072058 \\
\hline $\mathrm{H}$ & -1.981929 & -0.474367 & -3.257461 & $\mathrm{H}$ & & 1.122530 & -2.144126 & -2.851469 \\
\hline $\mathrm{H}$ & -1.381337 & -2.112721 & -2.881512 & $\mathrm{H}$ & & 0.736871 & -3.313802 & -1.563347 \\
\hline $\mathrm{C}$ & -3.359557 & 1.278801 & -1.310367 & C & & 2.713107 & 0.299105 & -2.479054 \\
\hline $\mathrm{H}$ & -4.250555 & 0.776185 & -1.728470 & $\mathrm{H}$ & & 3.621915 & -0.236419 & -2.811149 \\
\hline $\mathrm{H}$ & -3.722913 & 2.086149 & -0.658117 & $\mathrm{H}$ & & 2.954367 & 1.373361 & -2.488777 \\
\hline $\mathrm{H}$ & -2.825827 & 1.742901 & -2.156840 & $\mathrm{H}$ & & 1.944408 & 0.115577 & -3.250530 \\
\hline $\mathrm{C}$ & 2.343518 & 0.449257 & 2.639898 & C & & 3.073099 & 1.707123 & 1.511476 \\
\hline $\mathrm{H}$ & 3.403698 & 0.371434 & 2.944715 & $\mathrm{H}$ & & 4.174715 & 1.609863 & 1.500849 \\
\hline $\mathrm{H}$ & 2.050397 & 1.502313 & 2.788128 & $\mathrm{H}$ & & 2.836784 & 2.719138 & 1.146961 \\
\hline $\mathrm{H}$ & 1.762267 & -0.169248 & 3.342455 & $\mathrm{H}$ & & 2.756624 & 1.643856 & 2.564098 \\
\hline $\mathrm{C}$ & 3.148989 & 2.161652 & 0.046744 & $\mathrm{C}$ & & 2.955040 & 1.625236 & -1.730620 \\
\hline $\mathrm{H}$ & 2.886344 & 2.770397 & -0.833105 & $\mathrm{H}$ & & 2.271696 & 1.845909 & -2.570293 \\
\hline $\mathrm{H}$ & 2.889556 & 2.735670 & 0.949025 & $\mathrm{H}$ & & 3.175819 & 2.573001 & -1.216449 \\
\hline $\mathrm{H}$ & 4.249176 & 2.046932 & 0.040234 & $\mathrm{H}$ & & 3.901468 & 1.274832 & -2.181531 \\
\hline $\mathrm{C}$ & 1.818265 & -2.559069 & 1.668758 & $\mathrm{C}$ & & 2.077475 & -1.112085 & 2.612743 \\
\hline $\mathrm{H}$ & 1.279467 & -3.402249 & 1.213893 & $\mathrm{H}$ & & 1.492892 & -2.027681 & 2.789652 \\
\hline $\mathrm{H}$ & 2.845920 & -2.911903 & 1.876522 & $\mathrm{H}$ & & 3.116394 & -1.327475 & 2.924506 \\
\hline $\mathrm{H}$ & 1.357002 & -2.336998 & 2.643327 & $\mathrm{H}$ & & 1.693446 & -0.330166 & 3.288229 \\
\hline $\mathrm{C}$ & 1.917725 & -2.598133 & -1.571728 & $\mathrm{C}$ & & 1.800516 & -3.064491 & 0.059736 \\
\hline $\mathrm{H}$ & 1.402963 & -2.432924 & -2.532946 & $\mathrm{H}$ & & 1.227625 & -3.510587 & -0.766871 \\
\hline $\mathrm{H}$ & 2.955769 & -2.892061 & -1.813408 & $\mathrm{H}$ & & 2.837254 & -3.439426 & -0.035641 \\
\hline $\mathrm{H}$ & 1.446800 & -3.458892 & -1.076590 & $\mathrm{H}$ & & 1.408457 & -3.455148 & 1.008274 \\
\hline $\mathrm{C}$ & 2.677159 & 0.342631 & -2.557895 & C & & 2.062840 & -1.294098 & -2.602813 \\
\hline $\mathrm{H}$ & 2.163176 & -0.292740 & -3.296765 & $\mathrm{H}$ & & 1.407524 & -2.166478 & -2.745818 \\
\hline $\mathrm{H}$ & 2.432193 & 1.393554 & -2.781158 & $\mathrm{H}$ & & 1.779907 & -0.527778 & -3.344424 \\
\hline $\mathrm{H}$ & 3.763071 & 0.222080 & -2.730828 & $\mathrm{H}$ & & 3.089497 & -1.616591 & -2.859589 \\
\hline & II $\beta 1-I I F \beta 1]$ & & & & cture IIF $\alpha$ & & & \\
\hline $\mathrm{Zr}$ & 0.013152 & 0.145605 & -0.070300 & $\mathrm{Zr}$ & 0.009815 & $\begin{array}{ll}5 & 0.020\end{array}$ & $118 \quad-0.27$ & 7524 \\
\hline $\mathrm{C}$ & -2.407069 & 0.376151 & -1.050290 & C & 2.474059 & $\begin{array}{ll}9 & 0.750\end{array}$ & $615-0.63$ & 4954 \\
\hline $\mathrm{C}$ & -2.047567 & -1.023674 & -1.122233 & $\mathrm{C}$ & 2.089525 & $\begin{array}{ll}5 & 1.271\end{array}$ & $\begin{array}{ll}786 & 0.659\end{array}$ & 144 \\
\hline $\mathrm{C}$ & -1.964375 & -1.530441 & 0.238184 & $\mathrm{C}$ & 1.837895 & $\begin{array}{ll}5 & 0.144\end{array}$ & $\begin{array}{ll}728 & 1.537\end{array}$ & 277 \\
\hline
\end{tabular}




\begin{tabular}{|c|c|c|c|c|c|c|c|}
\hline $\mathrm{C}$ & -2.185408 & -0.426715 & 1.145264 & $\mathrm{C}$ & 2.140837 & -1.073506 & 0.799793 \\
\hline $\mathrm{C}$ & -2.453746 & 0.759863 & 0.346144 & $\mathrm{C}$ & 2.524367 & -0.700722 & -0.538331 \\
\hline $\mathrm{C}$ & 2.374953 & -0.207533 & 0.984472 & $\mathrm{C}$ & -2.609354 & -0.153126 & 0.141588 \\
\hline $\mathrm{C}$ & 1.599104 & -1.418059 & 1.170303 & $\mathrm{C}$ & -2.253379 & -1.163757 & -0.832655 \\
\hline $\mathrm{C}$ & 1.399619 & -2.015437 & -0.144354 & $\mathrm{C}$ & -1.385009 & -2.132048 & -0.184246 \\
\hline $\mathrm{C}$ & 2.010168 & -1.147053 & -1.132129 & $\mathrm{C}$ & -1.224357 & -1.722811 & 1.195357 \\
\hline $\mathrm{C}$ & 2.620823 & -0.038708 & -0.431781 & $\mathrm{C}$ & -1.943220 & -0.473026 & 1.383384 \\
\hline $\mathrm{C}$ & 0.268506 & 1.276878 & -2.024449 & $\mathrm{C}$ & -0.620669 & 2.167910 & -0.314520 \\
\hline $\mathrm{C}$ & 0.205681 & 2.556736 & -1.196811 & $\mathrm{C}$ & -1.174044 & 3.275634 & 0.591210 \\
\hline $\mathrm{H}$ & 1.243076 & 1.161509 & -2.512482 & $\mathrm{H}$ & 0.257665 & 2.567225 & -0.865820 \\
\hline $\mathrm{H}$ & -0.519297 & 1.225316 & -2.782184 & $\mathrm{H}$ & -1.397516 & 1.919920 & -1.081791 \\
\hline $\mathrm{H}$ & -0.829374 & 2.923016 & -1.111547 & $\mathrm{H}$ & -2.094793 & 2.926124 & 1.083582 \\
\hline $\mathrm{C}$ & 1.116599 & 3.720079 & -1.647439 & $\mathrm{C}$ & -1.474877 & 4.598395 & -0.146293 \\
\hline $\mathrm{H}$ & 2.170761 & 3.414202 & -1.699430 & $\mathrm{H}$ & -0.566596 & 5.008902 & -0.617953 \\
\hline $\mathrm{H}$ & 0.812217 & 4.048765 & -2.652968 & $\mathrm{H}$ & -2.226966 & 4.453039 & -0.939400 \\
\hline $\mathrm{H}$ & 1.037841 & 4.583764 & -0.967370 & $\mathrm{H}$ & -1.863724 & 5.359087 & 0.549835 \\
\hline $\mathrm{H}$ & 0.514480 & 2.363626 & -0.110291 & $\mathrm{H}$ & -0.450165 & 3.475587 & 1.398290 \\
\hline $\mathrm{C}$ & 0.034764 & 1.912492 & 3.088997 & $\mathrm{C}$ & 0.392969 & -0.672695 & -3.040649 \\
\hline $\mathrm{C}$ & 0.305859 & 2.950953 & 2.266686 & $\mathrm{C}$ & -0.288049 & 0.499144 & -3.137764 \\
\hline $\mathrm{H}$ & -0.491778 & 3.571981 & 1.848965 & $\mathrm{H}$ & 1.475927 & -0.706627 & -3.153164 \\
\hline $\mathrm{H}$ & 1.333554 & 3.259675 & 2.052820 & $\mathrm{H}$ & -0.126522 & -1.631506 & -2.957700 \\
\hline $\mathrm{H}$ & 0.832541 & 1.341652 & 3.568682 & $\mathrm{H}$ & 0.233966 & 1.446136 & -3.303960 \\
\hline $\mathrm{H}$ & -0.989244 & 1.657314 & 3.366934 & $\mathrm{H}$ & -1.380014 & 0.528222 & -3.138642 \\
\hline $\mathrm{C}$ & 3.583129 & 0.960078 & -1.028020 & $\mathrm{C}$ & -3.694753 & 0.880630 & -0.048724 \\
\hline $\mathrm{H}$ & 3.658412 & 1.881927 & -0.429887 & $\mathrm{H}$ & -3.535766 & 1.533546 & -0.924777 \\
\hline $\mathrm{H}$ & 4.595990 & 0.516392 & -1.065006 & $\mathrm{H}$ & -4.664806 & 0.373106 & -0.201231 \\
\hline $\mathrm{H}$ & 3.324838 & 1.238267 & -2.061966 & $\mathrm{H}$ & -3.803939 & 1.526618 & 0.833842 \\
\hline $\mathrm{C}$ & 3.086084 & 0.568370 & 2.071948 & $\mathrm{C}$ & -2.181633 & 0.203074 & 2.714917 \\
\hline $\mathrm{H}$ & 2.706271 & 0.324654 & 3.074926 & $\mathrm{H}$ & -2.231874 & 1.299625 & 2.629218 \\
\hline $\mathrm{H}$ & 4.158145 & 0.296904 & 2.065277 & $\mathrm{H}$ & -3.142984 & -0.135098 & 3.145154 \\
\hline $\mathrm{H}$ & 3.037984 & 1.660928 & 1.936574 & $\mathrm{H}$ & -1.398109 & -0.041978 & 3.447310 \\
\hline $\mathrm{C}$ & 1.331059 & -2.086631 & 2.501719 & $\mathrm{C}$ & -0.780609 & -2.631807 & 2.317972 \\
\hline $\mathrm{H}$ & 1.100496 & -1.362599 & 3.300683 & $\mathrm{H}$ & -1.678128 & -3.109936 & 2.754384 \\
\hline $\mathrm{H}$ & 0.496908 & -2.802163 & 2.449855 & $\mathrm{H}$ & -0.124114 & -3.440666 & 1.970512 \\
\hline $\mathrm{H}$ & 2.222467 & -2.653626 & 2.829084 & $\mathrm{H}$ & -0.276966 & -2.101279 & 3.139657 \\
\hline
\end{tabular}




\begin{tabular}{|c|c|c|c|c|c|c|c|c|c|}
\hline $\mathrm{C}$ & 1.036742 & -3.457788 & -0.416187 & $\mathrm{C}$ & -2.957643 & \multicolumn{2}{|c|}{-1.354906} & \multicolumn{2}{|c|}{-2.159795} \\
\hline $\mathrm{H}$ & 0.512692 & -3.928748 & 0.425356 & $\mathrm{H}$ & -2.320030 & \multicolumn{2}{|c|}{-1.789777} & -2.947 & 17092 \\
\hline $\mathrm{H}$ & 0.428693 & -3.589347 & -1.324898 & $\mathrm{H}$ & -3.803445 & \multicolumn{2}{|c|}{-2.054938} & -2.025 & 59997 \\
\hline $\mathrm{H}$ & 1.970418 & -4.029742 & -0.574772 & $\mathrm{H}$ & -3.386892 & \multicolumn{2}{|c|}{-0.415343} & -2.543 & 3560 \\
\hline $\mathrm{C}$ & 2.201249 & -1.493411 & -2.590103 & $\mathrm{C}$ & -0.969099 & \multicolumn{2}{|c|}{-3.470737} & -0.757 & 7338 \\
\hline $\mathrm{H}$ & 3.093177 & -2.136926 & -2.711525 & $\mathrm{H}$ & -1.044138 & \multicolumn{2}{|c|}{-3.496040} & -1.856 & 6627 \\
\hline $\mathrm{H}$ & 1.343404 & -2.046650 & -3.003753 & $\mathrm{H}$ & 0.060119 & \multicolumn{2}{|c|}{-3.753773} & -0.482 & 2154 \\
\hline $\mathrm{H}$ & 2.360678 & -0.602132 & -3.216557 & $\mathrm{H}$ & -1.633332 & \multicolumn{2}{|c|}{-4.269602} & -0.378 & 8212 \\
\hline $\mathrm{C}$ & -2.051402 & -2.980391 & 0.651333 & $\mathrm{C}$ & 2.992868 & \multicolumn{2}{|c|}{1.591505} & -1.781 & 1011 \\
\hline $\mathrm{H}$ & -1.503786 & -3.200192 & 1.580707 & $\mathrm{H}$ & 2.928982 & \multicolumn{2}{|c|}{1.079556} & -2.755 & 5072 \\
\hline $\mathrm{H}$ & -3.113956 & -3.223587 & 0.845173 & $\mathrm{H}$ & 2.462641 & \multicolumn{2}{|c|}{2.554746} & -1.868 & 8061 \\
\hline $\mathrm{H}$ & -1.706373 & -3.669700 & -0.130203 & $\mathrm{H}$ & 4.060529 & \multicolumn{2}{|c|}{1.831833} & -1.620 & 0302 \\
\hline $\mathrm{C}$ & -2.419443 & -0.589512 & 2.633356 & $\mathrm{C}$ & 2.234206 & \multicolumn{2}{|c|}{2.707993} & 1.097 & 7918 \\
\hline $\mathrm{H}$ & -2.743638 & 0.351691 & 3.102293 & $\mathrm{H}$ & 1.880274 & \multicolumn{2}{|c|}{3.429135} & 0.343 & 3224 \\
\hline $\mathrm{H}$ & -3.229577 & -1.321800 & 2.803568 & $\mathrm{H}$ & 1.701879 & \multicolumn{2}{|c|}{2.910822} & 2.038 & 8909 \\
\hline $\mathrm{H}$ & -1.536420 & -0.960174 & 3.180769 & $\mathrm{H}$ & 3.303502 & \multicolumn{2}{|c|}{2.929612} & 1.274 & 4980 \\
\hline $\mathrm{C}$ & -2.032804 & -1.849329 & -2.389325 & $\mathrm{C}$ & 1.633707 & \multicolumn{2}{|c|}{0.262044} & 3.031 & 1405 \\
\hline $\mathrm{H}$ & -1.536883 & -2.821121 & -2.246006 & $\mathrm{H}$ & 0.920436 & \multicolumn{2}{|c|}{1.058188} & 3.298 & 8717 \\
\hline $\mathrm{H}$ & -3.066343 & -2.060819 & -2.721131 & $\mathrm{H}$ & 1.281048 & \multicolumn{2}{|c|}{-0.676760} & 3.482 & 2311 \\
\hline $\mathrm{H}$ & -1.525440 & -1.330494 & -3.219293 & $\mathrm{H}$ & 2.593699 & \multicolumn{2}{|c|}{0.512158} & 3.520 & 0519 \\
\hline $\mathrm{C}$ & -2.920566 & 1.205343 & -2.201607 & $\mathrm{C}$ & 2.398041 & \multicolumn{2}{|c|}{-2.442720} & 1.385 & 5482 \\
\hline $\mathrm{H}$ & -2.553603 & 2.245596 & -2.196697 & $\mathrm{H}$ & 1.986125 & \multicolumn{2}{|c|}{-2.554908} & 2.396 & 6494 \\
\hline $\mathrm{H}$ & -2.672061 & 0.759799 & -3.176655 & $\mathrm{H}$ & 2.009534 & \multicolumn{2}{|c|}{-3.263270} & 0.760 & 0147 \\
\hline $\mathrm{H}$ & -4.023863 & 1.260552 & -2.145587 & $\mathrm{H}$ & 3.490976 & \multicolumn{2}{|c|}{-2.597288} & 1.465 & 5503 \\
\hline $\mathrm{C}$ & -3.009141 & 2.072726 & 0.855679 & $\mathrm{C}$ & 3.171854 & \multicolumn{2}{|c|}{-1.653900} & -1.521 & 1966 \\
\hline $\mathrm{H}$ & -4.113751 & 2.022732 & 0.885511 & $\mathrm{H}$ & 4.112988 & \multicolumn{2}{|c|}{-2.040363} & -1.088 & 8881 \\
\hline $\mathrm{H}$ & -2.673322 & 2.313016 & 1.875975 & $\mathrm{H}$ & 2.549281 & -2.534 & 129 & -1.756 & 6889 \\
\hline $\mathrm{H}$ & -2.749323 & 2.922427 & 0.202929 & $\mathrm{H}$ & 3.446846 & -1.1608 & 816 & -2.467 & 7552 \\
\hline & {$[\mathbf{I} \alpha 2-\mathbf{I I P} \gamma]^{\ddagger}$} & & & & acture $\mathbf{I I F} \boldsymbol{\alpha}$ & & & & \\
\hline $\mathrm{Zr}$ & -0.020635 & -0.141099 & -0.016047 & $\mathrm{Zr}$ & & 010749 & -0.32 & 22869 & -0.016888 \\
\hline $\mathrm{C}$ & -1.824516 & -1.241735 & -1.497415 & $\mathrm{C}$ & & 143995 & 0.42 & 27696 & -2.238407 \\
\hline $\mathrm{C}$ & -2.495729 & -0.197154 & -0.755653 & $\mathrm{C}$ & & 300429 & -0.70 & 04098 & -2.564156 \\
\hline $\mathrm{C}$ & -1.947143 & 1.078726 & -1.193474 & $\mathrm{C}$ & & 086113 & -0.28 & 287341 & -2.405459 \\
\hline $\mathrm{C}$ & -0.969666 & 0.822083 & -2.227605 & $\mathrm{C}$ & & 092620 & 1.08 & 80487 & -1.952279 \\
\hline $\mathrm{C}$ & -0.862658 & -0.618514 & -2.394857 & $\mathrm{C}$ & 0.2 & 288576 & 1.51 & 15901 & -1.810857 \\
\hline
\end{tabular}




\begin{tabular}{|c|c|c|c|c|c|c|c|}
\hline $\mathrm{C}$ & 2.354923 & -0.904672 & -0.525772 & $\mathrm{C}$ & 0.090331 & 1.262893 & 2.057865 \\
\hline C & 2.317293 & 0.473452 & -0.984171 & $\mathrm{C}$ & -1.219783 & 1.435921 & 1.450552 \\
\hline $\mathrm{C}$ & 2.067313 & 1.319091 & 0.161804 & $\mathrm{C}$ & -1.949903 & 0.194410 & 1.591363 \\
\hline $\mathrm{C}$ & 2.086109 & 0.471414 & 1.355210 & $\mathrm{C}$ & -1.115257 & -0.722380 & 2.351725 \\
\hline $\mathrm{C}$ & 2.283579 & -0.888687 & 0.933754 & $\mathrm{C}$ & 0.131596 & -0.055579 & 2.647731 \\
\hline $\mathrm{C}$ & -0.785552 & -1.560510 & 1.522472 & $\mathrm{C}$ & 2.137162 & -0.881931 & 0.358602 \\
\hline $\mathrm{C}$ & -0.551324 & -1.854763 & 3.017972 & $\mathrm{C}$ & 3.663827 & -0.737110 & 0.307927 \\
\hline $\mathrm{H}$ & -1.808583 & -1.879970 & 1.270745 & $\mathrm{H}$ & 1.861838 & -1.316703 & 1.342081 \\
\hline $\mathrm{H}$ & -0.118927 & -2.233970 & 0.880726 & $\mathrm{H}$ & 1.844727 & -1.620916 & -0.443994 \\
\hline $\mathrm{H}$ & -0.172804 & -2.884478 & 3.153437 & $\mathrm{H}$ & 4.125163 & -1.712353 & 0.558975 \\
\hline C & -1.843327 & -1.711927 & 3.845562 & $\mathrm{C}$ & 4.249257 & 0.329444 & 1.252497 \\
\hline $\mathrm{H}$ & -2.284961 & -0.707565 & 3.746983 & $\mathrm{H}$ & 3.854781 & 1.330744 & 1.019692 \\
\hline $\mathrm{H}$ & -2.601864 & -2.444443 & 3.524337 & $\mathrm{H}$ & 4.031082 & 0.107155 & 2.308853 \\
\hline $\mathrm{H}$ & -1.644179 & -1.886258 & 4.914836 & $\mathrm{H}$ & 5.345131 & 0.365574 & 1.146469 \\
\hline $\mathrm{H}$ & 0.221933 & -1.192389 & 3.440580 & $\mathrm{H}$ & 3.985284 & -0.514620 & -0.718628 \\
\hline $\mathrm{C}$ & -1.123063 & 3.338680 & 2.605894 & $\mathrm{C}$ & -1.360967 & -2.764176 & -0.364250 \\
\hline $\mathrm{C}$ & -1.434156 & 2.043140 & 2.822763 & $\mathrm{C}$ & -0.188513 & -3.185227 & 0.184879 \\
\hline $\mathrm{H}$ & -0.305785 & 3.828642 & 3.144937 & $\mathrm{H}$ & -2.191072 & -2.413442 & 0.253877 \\
\hline $\mathrm{H}$ & -1.699618 & 3.964592 & 1.918304 & $\mathrm{H}$ & -1.555659 & -2.883426 & -1.428885 \\
\hline $\mathrm{H}$ & -0.886039 & 1.436616 & 3.548671 & $\mathrm{H}$ & -0.025398 & -3.186900 & 1.264848 \\
\hline $\mathrm{H}$ & -2.292344 & 1.570002 & 2.335330 & $\mathrm{H}$ & 0.594189 & -3.634534 & -0.433253 \\
\hline C & -3.722404 & -0.373849 & 0.108040 & $\mathrm{C}$ & 0.773207 & -1.978425 & -3.231388 \\
\hline $\mathrm{H}$ & -3.751196 & -1.355422 & 0.606383 & $\mathrm{H}$ & 1.784003 & -2.273176 & -2.904890 \\
\hline H & -3.810878 & 0.400371 & 0.886541 & $\mathrm{H}$ & 0.099766 & -2.832592 & -3.052512 \\
\hline $\mathrm{H}$ & -4.630668 & -0.305928 & -0.519417 & $\mathrm{H}$ & 0.820053 & -1.837081 & -4.327336 \\
\hline $\mathrm{C}$ & -2.489882 & 2.446691 & -0.840132 & $\mathrm{C}$ & -2.312341 & -0.997892 & -2.940817 \\
\hline $\mathrm{H}$ & -3.010643 & 2.454130 & 0.129157 & $\mathrm{H}$ & -2.084946 & -2.011448 & -3.306230 \\
\hline $\mathrm{H}$ & -1.705351 & 3.220558 & -0.815636 & $\mathrm{H}$ & -3.135136 & -1.068366 & -2.209267 \\
\hline H & -3.227029 & 2.760948 & -1.602955 & $\mathrm{H}$ & -2.707824 & -0.436667 & -3.807651 \\
\hline C & -0.415875 & 1.883598 & -3.150166 & $\mathrm{C}$ & -2.311158 & 1.971380 & -2.017519 \\
\hline $\mathrm{H}$ & -1.221615 & 2.232334 & -3.823009 & $\mathrm{H}$ & -2.574796 & 2.132561 & -3.080338 \\
\hline $\mathrm{H}$ & -0.045883 & 2.773087 & -2.613611 & $\mathrm{H}$ & -3.200072 & 1.531754 & -1.537433 \\
\hline $\mathrm{H}$ & 0.393231 & 1.505297 & -3.788964 & $\mathrm{H}$ & -2.135055 & 2.962159 & -1.579839 \\
\hline $\mathrm{C}$ & -0.161271 & -1.337289 & -3.526491 & $\mathrm{C}$ & 0.771819 & 2.933361 & -1.602488 \\
\hline H & 0.196485 & -2.337690 & -3.235819 & $\mathrm{H}$ & 1.700015 & 2.976973 & -1.009957 \\
\hline
\end{tabular}




\begin{tabular}{|c|c|c|c|}
\hline $\mathrm{H}$ & -0.866586 & -1.480683 & -4.366183 \\
\hline $\mathrm{H}$ & 0.694604 & -0.770196 & -3.921022 \\
\hline $\mathrm{C}$ & -2.189908 & -2.708765 & -1.492085 \\
\hline $\mathrm{H}$ & -2.539370 & -3.055373 & -0.506739 \\
\hline $\mathrm{H}$ & -3.013306 & -2.889654 & -2.208056 \\
\hline $\mathrm{H}$ & -1.349623 & -3.352102 & -1.798662 \\
\hline $\mathrm{C}$ & 2.075806 & 2.834015 & 0.169482 \\
\hline $\mathrm{H}$ & 1.396390 & 3.253641 & 0.928812 \\
\hline $\mathrm{H}$ & 3.090313 & 3.208675 & 0.400917 \\
\hline $\mathrm{H}$ & 1.791830 & 3.256313 & -0.807363 \\
\hline $\mathrm{C}$ & 2.750118 & 0.945178 & -2.349849 \\
\hline $\mathrm{H}$ & 2.382263 & 1.952195 & -2.590226 \\
\hline $\mathrm{H}$ & 3.855609 & 0.994965 & -2.367678 \\
\hline $\mathrm{H}$ & 2.453171 & 0.260425 & -3.157983 \\
\hline $\mathrm{C}$ & 2.777262 & -2.104779 & -1.347426 \\
\hline $\mathrm{H}$ & 2.525847 & -1.992356 & -2.412316 \\
\hline $\mathrm{H}$ & 3.873832 & -2.236635 & -1.285613 \\
\hline $\mathrm{H}$ & 2.322724 & -3.042337 & -0.987031 \\
\hline $\mathrm{C}$ & 2.608874 & -2.074491 & 1.810173 \\
\hline $\mathrm{H}$ & 2.342803 & -1.904440 & 2.862333 \\
\hline $\mathrm{H}$ & 2.112660 & -3.002064 & 1.479246 \\
\hline $\mathrm{H}$ & 3.697814 & -2.264606 & 1.773935 \\
\hline $\mathrm{C}$ & 2.146444 & 0.988013 & 2.774063 \\
\hline $\mathrm{H}$ & 3.198536 & 1.200743 & 3.041890 \\
\hline $\mathrm{H}$ & 1.586006 & 1.926254 & 2.900256 \\
\hline $\mathrm{H}$ & 1.769120 & 0.261064 & 3.509255 \\
\hline
\end{tabular}

Structure IIFß1

$\begin{array}{llll}\text { Zr } & 0.010241 & 0.218771 & 0.093061 \\ \text { C } & -2.417452 & 0.656200 & -0.850242 \\ \text { C } & -1.985487 & -0.631640 & -1.349819 \\ \text { C } & -1.919934 & -1.545330 & -0.221113 \\ \text { C } & -2.242249 & -0.805877 & 0.975910 \\ \text { C } & -2.523991 & 0.569960 & 0.590433 \\ \text { C } & 2.461934 & -0.437317 & 0.909742 \\ \text { C } & 1.692652 & -1.648040 & 0.696040\end{array}$

\begin{tabular}{|c|c|c|c|}
\hline $\mathrm{H}$ & 0.994382 & 3.399148 & -2.581059 \\
\hline $\mathrm{H}$ & 0.021083 & 3.568530 & -1.110996 \\
\hline $\mathrm{C}$ & 2.596302 & 0.583052 & -2.616326 \\
\hline $\mathrm{H}$ & 3.120944 & -0.381564 & -2.688922 \\
\hline $\mathrm{H}$ & 2.656305 & 1.054762 & -3.615808 \\
\hline $\mathrm{H}$ & 3.150392 & 1.229988 & -1.919888 \\
\hline $\mathrm{C}$ & -3.423775 & 0.001667 & 1.301131 \\
\hline $\mathrm{H}$ & -3.716908 & -1.061056 & 1.306946 \\
\hline $\mathrm{H}$ & -4.032636 & 0.498966 & 2.079205 \\
\hline $\mathrm{H}$ & -3.729643 & 0.430034 & 0.333393 \\
\hline $\mathrm{C}$ & -1.843660 & 2.781858 & 1.162066 \\
\hline $\mathrm{H}$ & -2.780238 & 2.700983 & 0.595036 \\
\hline $\mathrm{H}$ & -2.092759 & 3.262612 & 2.127440 \\
\hline $\mathrm{H}$ & -1.170264 & 3.473188 & 0.633936 \\
\hline $\mathrm{C}$ & 1.081510 & 2.378813 & 2.298921 \\
\hline $\mathrm{H}$ & 1.125231 & 3.087588 & 1.457385 \\
\hline $\mathrm{H}$ & 0.786777 & 2.959402 & 3.193750 \\
\hline $\mathrm{H}$ & 2.098684 & 2.000796 & 2.475215 \\
\hline $\mathrm{C}$ & 1.184839 & -0.568973 & 3.600128 \\
\hline $\mathrm{H}$ & 1.390669 & -1.647620 & 3.482996 \\
\hline $\mathrm{H}$ & 2.137144 & -0.030541 & 3.491867 \\
\hline $\mathrm{H}$ & 0.845477 & -0.422995 & 4.642415 \\
\hline $\mathrm{C}$ & -1.590062 & -1.996061 & 3.019421 \\
\hline $\mathrm{H}$ & -2.188167 & -1.735992 & 3.912650 \\
\hline $\mathrm{H}$ & -2.240020 & -2.621453 & 2.385693 \\
\hline $\mathrm{H}$ & -0.754691 & -2.620835 & 3.374513 \\
\hline
\end{tabular}

Structure IIP $\boldsymbol{\gamma}$

\begin{tabular}{|c|c|c|c|}
\hline $\mathrm{Zr}$ & -0.039021 & -0.007913 & -0.172183 \\
\hline $\mathrm{C}$ & -0.090415 & -2.604920 & -0.439871 \\
\hline $\mathrm{C}$ & -0.137058 & -2.304322 & 0.979884 \\
\hline $\mathrm{C}$ & -1.396594 & -1.648727 & 1.261008 \\
\hline $\mathrm{C}$ & -2.118322 & -1.516359 & 0.003339 \\
\hline $\mathrm{C}$ & -1.306413 & -2.108417 & -1.043451 \\
\hline $\mathrm{C}$ & 0.720304 & 2.411422 & 0.289559 \\
\hline $\mathrm{C}$ & -0.087920 & 2.495951 & -0.910139 \\
\hline
\end{tabular}




\begin{tabular}{|c|c|c|c|c|c|c|c|}
\hline $\mathrm{C}$ & 1.450492 & -1.765963 & -0.734195 & $\mathrm{C}$ & -1.442624 & 2.121013 & -0.560910 \\
\hline $\mathrm{C}$ & 2.006326 & -0.599586 & -1.384401 & $\mathrm{C}$ & -1.487272 & 1.877740 & 0.873355 \\
\hline $\mathrm{C}$ & 2.632407 & 0.220914 & -0.365687 & $\mathrm{C}$ & -0.143957 & 2.011927 & 1.390393 \\
\hline $\mathrm{C}$ & 0.207984 & 1.884064 & -1.503466 & $\mathrm{C}$ & 2.651721 & -0.456471 & -0.354573 \\
\hline $\mathrm{C}$ & 0.034881 & 2.878211 & -0.383669 & $\mathrm{C}$ & 4.154147 & -0.219183 & -0.068826 \\
\hline $\mathrm{H}$ & 1.200343 & 1.941282 & -1.960950 & $\mathrm{H}$ & 2.138462 & 0.054818 & 0.517073 \\
\hline $\mathrm{H}$ & -0.561636 & 1.936271 & -2.277351 & $\mathrm{H}$ & 2.447088 & -1.534287 & -0.273697 \\
\hline $\mathrm{H}$ & -1.007521 & 3.221526 & -0.302677 & $\mathrm{H}$ & 4.720709 & -0.718713 & -0.874175 \\
\hline $\mathrm{C}$ & 0.986138 & 4.093100 & -0.351123 & C & 4.624252 & -0.743176 & 1.297122 \\
\hline $\mathrm{H}$ & 2.041193 & 3.787485 & -0.379154 & $\mathrm{H}$ & 4.095423 & -0.248781 & 2.129572 \\
\hline $\mathrm{H}$ & 0.796750 & 4.722706 & -1.234274 & $\mathrm{H}$ & 4.465813 & -1.829828 & 1.392251 \\
\hline $\mathrm{H}$ & 0.826534 & 4.710450 & 0.547730 & $\mathrm{H}$ & 5.699964 & -0.554259 & 1.433459 \\
\hline $\mathrm{H}$ & 0.203093 & 2.373249 & 0.642017 & $\mathrm{H}$ & 4.375601 & 0.857379 & -0.157380 \\
\hline $\mathrm{C}$ & -0.064301 & 0.387998 & 2.917227 & $\mathrm{C}$ & 0.760926 & -0.090711 & -2.256100 \\
\hline $\mathrm{C}$ & 0.411692 & 1.635083 & 2.640427 & $\mathrm{C}$ & 2.236297 & 0.084988 & -1.762947 \\
\hline $\mathrm{H}$ & -0.245454 & 2.507300 & 2.708315 & $\mathrm{H}$ & 0.636888 & -1.065399 & -2.746459 \\
\hline $\mathrm{H}$ & 1.476919 & 1.827784 & 2.494553 & $\mathrm{H}$ & 0.525629 & 0.686282 & -2.993398 \\
\hline $\mathrm{H}$ & 0.594695 & -0.476503 & 3.004254 & $\mathrm{H}$ & 2.895481 & -0.455659 & -2.466311 \\
\hline $\mathrm{H}$ & -1.101640 & 0.244956 & 3.211100 & $\mathrm{H}$ & 2.532494 & 1.143188 & -1.813774 \\
\hline $\mathrm{C}$ & 3.560991 & 1.383486 & -0.630258 & $\mathrm{C}$ & 0.325813 & 3.125029 & -2.217146 \\
\hline $\mathrm{H}$ & 3.632675 & 2.076860 & 0.223047 & $\mathrm{H}$ & -0.340949 & 2.837021 & -3.044556 \\
\hline $\mathrm{H}$ & 4.582119 & 1.003480 & -0.821686 & $\mathrm{H}$ & 0.273314 & 4.226466 & -2.129202 \\
\hline $\mathrm{H}$ & 3.271064 & 1.965442 & -1.519097 & $\mathrm{H}$ & 1.359056 & 2.878784 & -2.510985 \\
\hline $\mathrm{C}$ & 3.233300 & -0.093373 & 2.166067 & $\mathrm{C}$ & 2.123223 & 2.956534 & 0.449378 \\
\hline $\mathrm{H}$ & 2.684543 & -0.288739 & 3.101294 & $\mathrm{H}$ & 2.702896 & 2.918777 & -0.485877 \\
\hline $\mathrm{H}$ & 4.145862 & -0.717062 & 2.207230 & $\mathrm{H}$ & 2.069211 & 4.020304 & 0.747311 \\
\hline $\mathrm{H}$ & 3.571570 & 0.954862 & 2.179943 & $\mathrm{H}$ & 2.698673 & 2.438305 & 1.233611 \\
\hline $\mathrm{C}$ & 1.507381 & -2.760774 & 1.706904 & C & 0.259123 & 1.977538 & 2.849294 \\
\hline $\mathrm{H}$ & 1.634543 & -2.410151 & 2.744216 & $\mathrm{H}$ & 0.194882 & 2.991528 & 3.286686 \\
\hline $\mathrm{H}$ & 0.521233 & -3.246405 & 1.635292 & $\mathrm{H}$ & -0.397089 & 1.328014 & 3.449497 \\
\hline $\mathrm{H}$ & 2.266819 & -3.549024 & 1.548182 & $\mathrm{H}$ & 1.297958 & 1.635890 & 2.993465 \\
\hline $\mathrm{C}$ & 1.120007 & -3.050919 & -1.458643 & $\mathrm{C}$ & -2.740123 & 1.850006 & 1.715875 \\
\hline $\mathrm{H}$ & 0.625869 & -3.786107 & -0.810663 & $\mathrm{H}$ & -3.587462 & 1.360550 & 1.215593 \\
\hline $\mathrm{H}$ & 0.500317 & -2.899659 & -2.355752 & $\mathrm{H}$ & -2.585157 & 1.370938 & 2.692879 \\
\hline $\mathrm{H}$ & 2.066465 & -3.509715 & -1.803071 & $\mathrm{H}$ & -3.050062 & 2.893014 & 1.917223 \\
\hline
\end{tabular}




\begin{tabular}{|c|c|c|c|}
\hline $\mathrm{C}$ & 2.171193 & -0.445830 & -2.878557 \\
\hline $\mathrm{H}$ & 3.054474 & -1.018742 & -3.219465 \\
\hline $\mathrm{H}$ & 1.301850 & -0.827592 & -3.436611 \\
\hline $\mathrm{H}$ & 2.333011 & 0.599875 & -3.182163 \\
\hline $\mathrm{C}$ & -1.953154 & -3.054152 & -0.294336 \\
\hline $\mathrm{H}$ & -1.378879 & -3.542607 & 0.508644 \\
\hline $\mathrm{H}$ & -3.002525 & -3.387466 & -0.178846 \\
\hline $\mathrm{H}$ & -1.601398 & -3.442118 & -1.258732 \\
\hline $\mathrm{C}$ & -2.604340 & -1.468202 & 2.290119 \\
\hline $\mathrm{H}$ & -2.939013 & -0.742351 & 3.047686 \\
\hline $\mathrm{H}$ & -3.452488 & -2.158615 & 2.127108 \\
\hline $\mathrm{H}$ & -1.786638 & -2.068813 & 2.723105 \\
\hline $\mathrm{C}$ & -1.915722 & -1.005102 & -2.813152 \\
\hline $\mathrm{H}$ & -1.415855 & -1.971867 & -2.972591 \\
\hline $\mathrm{H}$ & -2.934907 & -1.097021 & -3.232773 \\
\hline $\mathrm{H}$ & -1.385928 & -0.247547 & -3.414036 \\
\hline $\mathrm{C}$ & -2.968649 & 1.772400 & -1.704897 \\
\hline $\mathrm{H}$ & -2.695096 & 2.781388 & -1.355662 \\
\hline $\mathrm{H}$ & -2.664103 & 1.680256 & -2.758257 \\
\hline $\mathrm{H}$ & -4.073680 & 1.724045 & -1.687233 \\
\hline $\mathrm{C}$ & -3.137499 & 1.634813 & 1.474437 \\
\hline $\mathrm{H}$ & -4.237374 & 1.519402 & 1.498457 \\
\hline $\mathrm{H}$ & -2.791884 & 1.581077 & 2.519634 \\
\hline $\mathrm{H}$ & -2.933174 & 2.652798 & 1.104317 \\
\hline
\end{tabular}

\begin{tabular}{|c|c|c|c|}
\hline $\mathrm{C}$ & -2.634874 & 2.238016 & -1.484988 \\
\hline $\mathrm{H}$ & -3.529506 & 1.750814 & -1.070598 \\
\hline $\mathrm{H}$ & -2.890022 & 3.302516 & -1.642150 \\
\hline $\mathrm{H}$ & -2.439730 & 1.804436 & -2.480131 \\
\hline $\mathrm{C}$ & -3.584298 & -1.183717 & -0.164799 \\
\hline $\mathrm{H}$ & -4.161034 & -2.123400 & -0.252827 \\
\hline $\mathrm{H}$ & -3.996653 & -0.637747 & 0.695002 \\
\hline $\mathrm{H}$ & -3.788397 & -0.600715 & -1.076808 \\
\hline $\mathrm{C}$ & -1.957142 & -1.450642 & 2.651746 \\
\hline $\mathrm{H}$ & -2.880595 & -0.856026 & 2.648087 \\
\hline $\mathrm{H}$ & -2.209248 & -2.434604 & 3.088981 \\
\hline $\mathrm{H}$ & -1.242985 & -0.971726 & 3.342503 \\
\hline $\mathrm{C}$ & -1.769317 & -2.377254 & -2.455745 \\
\hline $\mathrm{H}$ & -2.396371 & -3.288661 & -2.478817 \\
\hline $\mathrm{H}$ & -2.378749 & -1.554596 & -2.863034 \\
\hline $\mathrm{H}$ & -0.928957 & -2.548532 & -3.146176 \\
\hline $\mathrm{C}$ & 0.927826 & -3.477086 & -1.137904 \\
\hline $\mathrm{H}$ & 1.299561 & -3.040311 & -2.081165 \\
\hline $\mathrm{H}$ & 1.795510 & -3.702238 & -0.497742 \\
\hline $\mathrm{H}$ & 0.467649 & -4.447298 & -1.399415 \\
\hline $\mathrm{C}$ & 0.843402 & -2.773488 & 2.033695 \\
\hline $\mathrm{H}$ & 0.891231 & -2.088345 & 2.896356 \\
\hline $\mathrm{H}$ & 0.530032 & -3.759145 & 2.425945 \\
\hline $\mathrm{H}$ & 1.866044 & -2.896373 & 1.643017 \\
\hline
\end{tabular}

Structure [II $\alpha 2-I I F \alpha 1]^{\neq}$

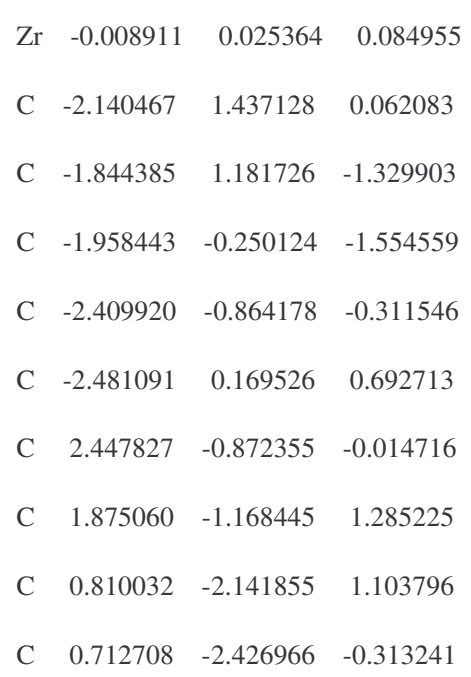

\begin{tabular}{|cccc}
\multicolumn{4}{|l}{ Structure [IIF $\alpha 1-$ IIP $\gamma]^{\neq}$} \\
Zr & 0.008471 & 0.102273 & -0.190260 \\
C & 2.555501 & 0.405009 & -0.855156 \\
C & 2.374792 & 1.218963 & 0.327741 \\
C & 2.037640 & 0.349122 & 1.428411 \\
C & 2.068988 & -1.022253 & 0.936348 \\
C & 2.359444 & -0.985122 & -0.481399 \\
C & -2.624294 & -0.298518 & -0.227568 \\
C & -2.038241 & -1.457654 & -0.875633 \\
C & -1.229915 & -2.163441 & 0.087647 \\
C & -1.318092 & -1.439873 & 1.349924
\end{tabular} 


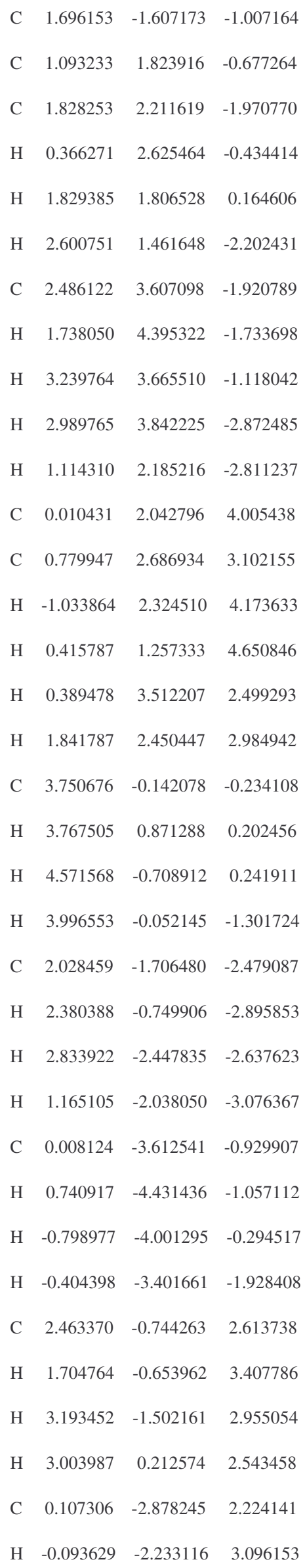

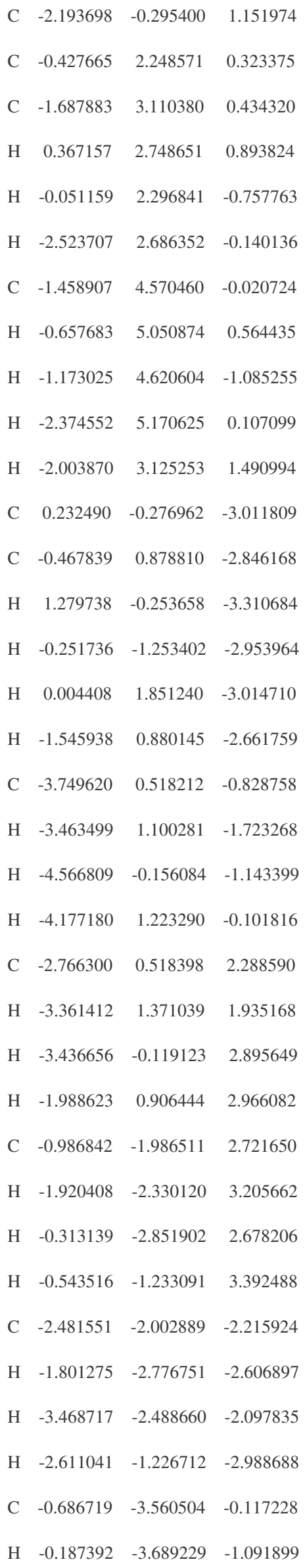




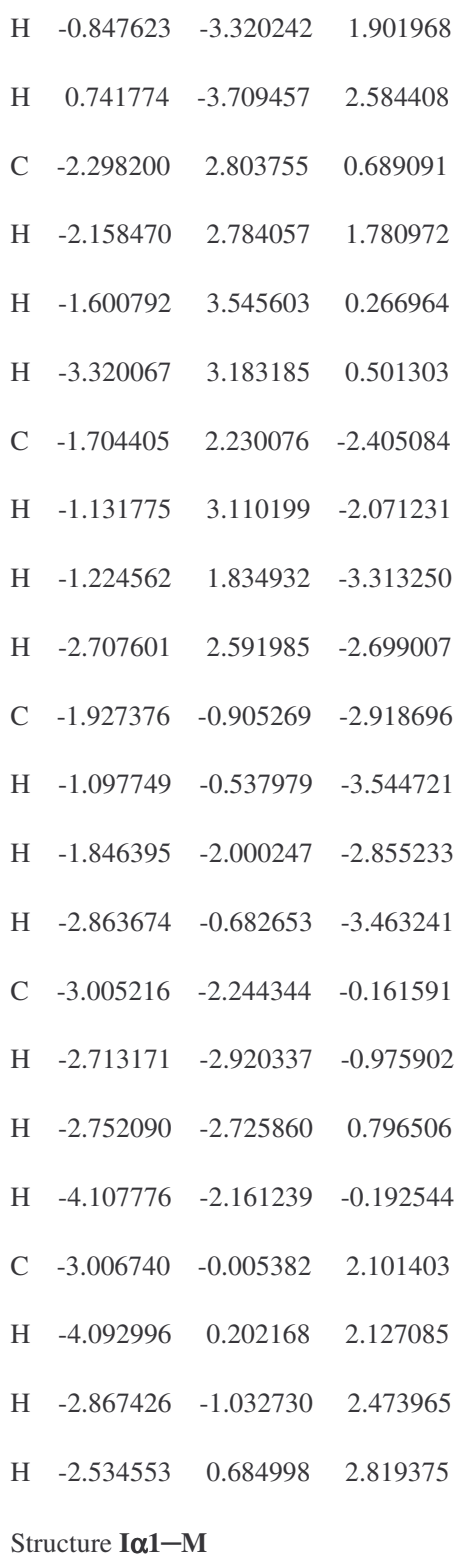

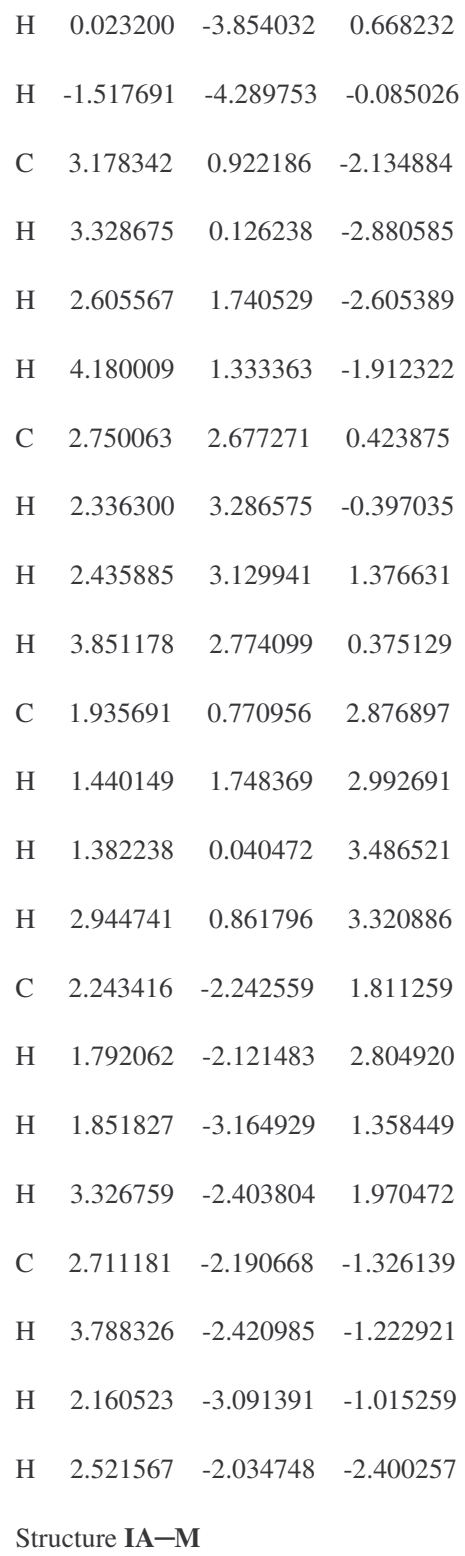




\begin{tabular}{|c|c|c|c|}
\hline $\mathrm{C}$ & -0.711273 & -2.699011 & -0.200460 \\
\hline $\mathrm{C}$ & -1.799871 & 3.455492 & -1.303435 \\
\hline $\mathrm{C}$ & 3.779864 & 0.943576 & -2.961729 \\
\hline $\mathrm{C}$ & 0.263684 & 2.606575 & -6.167540 \\
\hline $\mathrm{C}$ & 1.678256 & -3.147699 & -4.319264 \\
\hline $\mathrm{C}$ & -4.046629 & -0.913740 & -3.454333 \\
\hline $\mathrm{Al}$ & 2.390773 & -0.436884 & 0.153168 \\
\hline $\mathrm{H}$ & -1.130529 & 3.838280 & -0.509294 \\
\hline $\mathrm{H}$ & -2.787991 & 3.258736 & -0.849191 \\
\hline $\mathrm{H}$ & -1.922877 & 4.271950 & -2.039751 \\
\hline $\mathrm{H}$ & 4.085588 & 1.867715 & -2.440384 \\
\hline $\mathrm{H}$ & 4.020375 & 1.068570 & -4.035066 \\
\hline $\mathrm{H}$ & 4.412864 & 0.118701 & -2.584176 \\
\hline $\mathrm{H}$ & -0.122480 & 2.105643 & -7.074899 \\
\hline $\mathrm{H}$ & 1.327256 & 2.848299 & -6.349046 \\
\hline $\mathrm{H}$ & -0.286628 & 3.558910 & -6.053670 \\
\hline $\mathrm{H}$ & 2.466464 & -3.129825 & -5.097600 \\
\hline $\mathrm{H}$ & 1.204203 & -4.148425 & -4.359588 \\
\hline $\mathrm{H}$ & 2.188772 & -3.069464 & -3.338932 \\
\hline $\mathrm{H}$ & -4.214312 & -1.266551 & -4.489125 \\
\hline $\mathrm{H}$ & -4.744217 & -0.075287 & -3.272214 \\
\hline $\mathrm{H}$ & -4.322589 & -1.740751 & -2.774059 \\
\hline $\mathrm{H}$ & -1.292836 & -2.400839 & 0.692301 \\
\hline $\mathrm{H}$ & -1.325462 & -3.436502 & -0.752532 \\
\hline $\mathrm{C}$ & 0.824179 & -0.236408 & 1.928784 \\
\hline $\mathrm{H}$ & 0.196276 & -3.231989 & 0.138366 \\
\hline $\mathrm{C}$ & 3.342550 & 1.280729 & 0.449786 \\
\hline $\mathrm{C}$ & 3.281448 & -2.175681 & 0.482874 \\
\hline $\mathrm{Zr}$ & -0.511818 & -0.081487 & 3.868118 \\
\hline $\mathrm{C}$ & -1.830469 & 1.850695 & 2.710460 \\
\hline $\mathrm{C}$ & 0.662247 & 1.591632 & 4.908460 \\
\hline $\mathrm{C}$ & -2.250307 & 0.634156 & 2.066006 \\
\hline $\mathrm{C}$ & -2.928532 & -0.169984 & 3.046193 \\
\hline $\mathrm{C}$ & 1.113762 & -1.768949 & 5.010452 \\
\hline $\mathrm{C}$ & 0.217105 & -1.291298 & 6.028954 \\
\hline
\end{tabular}

\begin{tabular}{|c|c|c|c|}
\hline $\mathrm{C}$ & 0.233782 & -2.986449 & -0.357584 \\
\hline $\mathrm{C}$ & -2.570645 & 2.710049 & -0.867481 \\
\hline $\mathrm{C}$ & 3.333389 & 1.936892 & -3.078949 \\
\hline $\mathrm{C}$ & -0.730135 & 2.750237 & -5.896643 \\
\hline $\mathrm{C}$ & 2.299406 & -2.504871 & -4.618357 \\
\hline $\mathrm{C}$ & -3.717871 & -1.960991 & -3.209302 \\
\hline $\mathrm{Al}$ & 2.528834 & 0.041134 & 0.105916 \\
\hline $\mathrm{H}$ & -2.004100 & 3.141496 & -0.019939 \\
\hline $\mathrm{H}$ & -3.481957 & 2.232898 & -0.462065 \\
\hline $\mathrm{H}$ & -2.888692 & 3.556479 & -1.504600 \\
\hline $\mathrm{H}$ & 3.442910 & 2.867237 & -2.494556 \\
\hline $\mathrm{H}$ & 3.414196 & 2.201345 & -4.150826 \\
\hline $\mathrm{H}$ & 4.194796 & 1.285233 & -2.840690 \\
\hline $\mathrm{H}$ & -1.015297 & 2.216711 & -6.822530 \\
\hline $\mathrm{H}$ & 0.205964 & 3.300482 & -6.105433 \\
\hline $\mathrm{H}$ & -1.520874 & 3.493445 & -5.683882 \\
\hline $\mathrm{H}$ & 2.986962 & -2.203362 & -5.433235 \\
\hline $\mathrm{H}$ & 2.106092 & -3.589004 & -4.740825 \\
\hline $\mathrm{H}$ & 2.851302 & -2.378784 & -3.665815 \\
\hline $\mathrm{H}$ & -3.887354 & -2.246658 & -4.264368 \\
\hline $\mathrm{H}$ & -4.592973 & -1.370091 & -2.880908 \\
\hline $\mathrm{H}$ & -3.692767 & -2.892477 & -2.614115 \\
\hline $\mathrm{H}$ & 0.512950 & -2.862892 & 0.703154 \\
\hline $\mathrm{H}$ & -0.740233 & -3.513407 & -0.387059 \\
\hline C & 0.924768 & 0.043014 & 1.870704 \\
\hline $\mathrm{H}$ & 0.977786 & -3.665877 & -0.815818 \\
\hline $\mathrm{C}$ & 3.266180 & 1.871205 & 0.345213 \\
\hline $\mathrm{C}$ & 3.664633 & -1.539937 & 0.473630 \\
\hline $\mathrm{Zr}$ & -0.291219 & 0.079132 & 3.999045 \\
\hline $\mathrm{C}$ & -2.676833 & 0.397571 & 3.004821 \\
\hline $\mathrm{C}$ & -0.986078 & 2.048394 & 5.330208 \\
\hline C & -2.045244 & -0.580691 & 2.167592 \\
\hline C & -1.774119 & -1.742312 & 2.971320 \\
\hline $\mathrm{C}$ & 2.107227 & -0.114223 & 5.008741 \\
\hline & 1.215816 & 0.014285 & 6.12976 \\
\hline
\end{tabular}




\begin{tabular}{|c|c|c|c|}
\hline C & -1.107614 & -1.745304 & 5.698761 \\
\hline C & -2.938283 & 0.558578 & 4.294441 \\
\hline $\mathrm{C}$ & -2.264052 & 1.811339 & 4.080676 \\
\hline C & -1.025950 & -2.509790 & 4.475458 \\
\hline $\mathrm{C}$ & 0.349769 & -2.528864 & 4.058479 \\
\hline C & 2.153844 & 1.404764 & 5.258384 \\
\hline $\mathrm{H}$ & 0.578332 & 2.405194 & 4.156152 \\
\hline $\mathrm{H}$ & -3.424061 & 0.243927 & 5.215582 \\
\hline $\mathrm{C}$ & -0.747061 & -1.664160 & -6.299066 \\
\hline H & -1.501163 & -0.852562 & -6.365933 \\
\hline $\mathrm{H}$ & -0.086891 & -1.554587 & -7.182742 \\
\hline $\mathrm{H}$ & -1.297786 & -2.617602 & -6.427565 \\
\hline $\mathrm{H}$ & 0.744034 & -3.040882 & 3.183286 \\
\hline H & 2.187037 & -1.594002 & 4.973761 \\
\hline H & 0.492516 & -0.706926 & 6.903738 \\
\hline $\mathrm{H}$ & -1.294018 & 2.670882 & 2.236232 \\
\hline $\mathrm{H}$ & -2.120787 & 2.596428 & 4.819654 \\
\hline $\mathrm{H}$ & 3.862589 & -2.164505 & 1.422448 \\
\hline $\mathrm{H}$ & 2.598533 & -3.040671 & 0.526622 \\
\hline $\mathrm{H}$ & 4.001729 & -2.387688 & -0.327658 \\
\hline $\mathrm{H}$ & 3.552816 & 1.411589 & 1.527209 \\
\hline $\mathrm{H}$ & 4.317212 & 1.303951 & -0.064821 \\
\hline $\mathrm{H}$ & 2.785295 & 2.175669 & 0.122401 \\
\hline $\mathrm{H}$ & 0.698658 & 0.749557 & 1.464823 \\
\hline $\mathrm{H}$ & 0.378839 & -1.091447 & 1.402253 \\
\hline $\mathrm{H}$ & -2.106314 & 0.382954 & 1.015852 \\
\hline H & 1.793302 & -0.423043 & 2.412466 \\
\hline H & -3.402034 & -1.132642 & 2.864296 \\
\hline $\mathrm{H}$ & -2.002957 & -1.590585 & 6.296733 \\
\hline $\mathrm{H}$ & -1.848953 & -3.027891 & 3.987133 \\
\hline $\mathrm{H}$ & 0.124014 & 1.974051 & 5.797925 \\
\hline $\mathrm{H}$ & 2.264553 & 0.693693 & 6.095897 \\
\hline C & 2.856156 & 2.725953 & 5.641300 \\
\hline $\mathrm{H}$ & 2.813929 & 3.451487 & 4.811890 \\
\hline & 2.369735 & 3.191112 & 6.515310 \\
\hline
\end{tabular}

\begin{tabular}{|c|c|c|c|}
\hline $\mathrm{C}$ & 0.358474 & -1.136433 & 6.154927 \\
\hline $\mathrm{C}$ & -2.289809 & -1.496837 & 4.297850 \\
\hline $\mathrm{C}$ & -2.841395 & -0.174401 & 4.322973 \\
\hline $\mathrm{C}$ & 0.752345 & -2.004175 & 5.067372 \\
\hline C & 1.831489 & -1.374258 & 4.364547 \\
\hline $\mathrm{C}$ & 0.119123 & 2.537438 & 4.573275 \\
\hline $\mathrm{H}$ & -1.986454 & 2.252125 & 4.940223 \\
\hline $\mathrm{H}$ & -2.290443 & -2.202950 & 5.125357 \\
\hline $\mathrm{C}$ & -0.593708 & -1.602075 & -6.290092 \\
\hline $\mathrm{H}$ & -1.518053 & -0.988632 & -6.255405 \\
\hline $\mathrm{H}$ & -0.049504 & -1.299450 & -7.207199 \\
\hline $\mathrm{H}$ & -0.916745 & -2.651601 & -6.441595 \\
\hline $\mathrm{H}$ & 2.363528 & -1.786303 & 3.510476 \\
\hline $\mathrm{H}$ & 2.888913 & 0.589230 & 4.727472 \\
\hline H & 1.197433 & 0.833990 & 6.843767 \\
\hline H & -3.032124 & 1.375162 & 2.683911 \\
\hline H & -3.335552 & 0.292803 & 5.171662 \\
\hline $\mathrm{H}$ & 4.203972 & -1.442360 & 1.432983 \\
\hline $\mathrm{H}$ & 3.121119 & -2.498551 & 0.489798 \\
\hline $\mathrm{H}$ & 4.438842 & -1.629653 & -0.309986 \\
\hline $\mathrm{H}$ & 3.611078 & 1.998064 & 1.388778 \\
\hline $\mathrm{H}$ & 4.146717 & 2.048421 & -0.293631 \\
\hline $\mathrm{H}$ & 2.551843 & 2.687224 & 0.140338 \\
\hline $\mathrm{H}$ & 0.389576 & 0.709973 & 1.184354 \\
\hline $\mathrm{H}$ & 0.901401 & -1.020955 & 1.606039 \\
\hline $\mathrm{H}$ & -1.831640 & -0.474220 & 1.105489 \\
\hline $\mathrm{H}$ & 1.887010 & 0.430743 & 2.228884 \\
\hline $\mathrm{H}$ & -1.322238 & -2.667263 & 2.618603 \\
\hline $\mathrm{H}$ & -0.413697 & -1.340857 & 6.894154 \\
\hline $\mathrm{H}$ & 0.331980 & -2.982137 & 4.843510 \\
\hline $\mathrm{H}$ & -0.919229 & 2.043453 & 6.420848 \\
\hline $\mathrm{H}$ & 1.076260 & 2.688834 & 5.087204 \\
\hline $\mathrm{C}$ & 0.145486 & 2.534646 & 3.178363 \\
\hline $\mathrm{H}$ & -0.788532 & 2.553114 & 2.607403 \\
\hline & 1.052044 & 2.808405 & 2.637108 \\
\hline
\end{tabular}


$\begin{array}{llll}\mathrm{H} & 3.918270 & 2.560995 & 5.891376\end{array}$

$\begin{array}{llll}\mathrm{H} & 2.700315 & 0.957626 & 4.406676\end{array}$ 